\title{
Optimization of a complex flexible multibody systems with composite materials
}

\author{
Jorge A.C. Ambrósio • Maria Augusta Neto • \\ Rogério P. Leal
}

Received: 18 March 2007 / Accepted: 24 June 2007 / Published online: 25 July 2007

(C) Springer Science+Business Media B.V. 2007

\begin{abstract}
The paper presents a general optimization methodology for flexible multibody systems which is demonstrated to find optimal layouts of fiber composite structures components. The goal of the optimization process is to minimize the structural deformation and, simultaneously, to fulfill a set of multidisciplinary constraints, by finding the optimal values for the fiber orientation of composite structures. In this work, a general formulation for the computation of the first order analytical sensitivities based on the use of automatic differentiation tools is applied. A critical overview on the use of the sensitivities obtained by automatic differentiation against analytical sensitivities derived and implemented by hand is made with the purpose of identifying shortcomings and proposing solutions. The equations of motion and sensitivities of the flexible multibody system are solved simultaneously being the accelerations and velocities of the system and the sensitivities of the accelerations and of the velocities integrated in time using a multi-step multi-order integration algorithm. Then, the optimal design of the flexible multibody system is formulated to minimize the deformation energy of the system subjected to a set of technological and functional constraints. The methodologies proposed are first discussed for a simple demonstrative example and applied after to the optimization of a complex flexible multibody system, represented by a satellite antenna that is unfolded from its launching configuration to its functional state.
\end{abstract}

Keywords Optimization methods · Objective functions - Sensitivity analysis - Automatic differentiation · Flexible multibody dynamics $\cdot$ Composite finite elements

J.A.C. Ambrósio ( $₫)$

IDMEC, Instituto Superior Técnico, Technical University of Lisbon, Av. Rovisco Pais, 1, 1049-001

Lisboa, Portugal

e-mail: jorge@ dem.ist.utl.pt

M.A. Neto - R.P. Leal

Department of Mechanical Engineering, Faculdade de Ciência e Tecnologia da Universidade de Coimbra, 3020 Coimbra, Portugal

M.A. Neto

e-mail: augusta.neto@dem.uc.pt

R.P. Leal

e-mail: rogerio.leal@dem.uc.pt 


\section{Introduction}

The use of new materials in the production of light mechanisms and structural components not only puts new challenges to the analysis tools but also offers a large potential for more advanced designs. Methodologies for the analysis of flexible multibody systems made of composite materials constitute efficient numerical tools for the analysis and design of such systems. The use of optimization methodologies, in the framework of the analysis of flexible multibody systems, provide the necessary reanalysis tools that not only allow for the final designs to be closer to the production product but also ensure that such product is the best possible for selected functional goals. Optimization involves, in general, iterative schemes where the cost function and constraints are obtained in an analysis step and the sensitivities are computed to be used in the decision step of the optimization procedure. The computation of the sensitivity of a structural response to changes in design variables is often the major computational cost of the optimization process, and therefore, efficient algorithms for evaluating these sensitivities are of fundamental importance. Therefore, the efficient and reliable design methodology requires that (1) the analysis tool is able to represent the complex mechanics of the system, (2) the optimization methodology allows for finding the best design for suitable optimal criteria and design constraints, and (3) the evaluation of the sensitivities required for the optimization are accurate and cheap from the computational point of view.

Both nonlinear finite elements [1, 2] and flexible multibody dynamics [3-5] methodologies can be used to describe complex mechanisms for which the deformation plays a major role. When the mechanical components experience generalized nonlinear material deformations the use of nonlinear finite elements is basically unavoidable. However, if the deformations are elastic and moderate, when referred to a body fixed coordinate frame, or to a co-rotational frame, the use of flexible multibody dynamics methods leads to computational tools as general as the nonlinear finite element based tools, but more efficient from the computational point of view [6]. The type of applications foreseen for the methodologies proposed in this work involves the study of mechanisms for which the components are either rigid or flexible, but experiencing only linear elastic deformations. Therefore, flexible multibody models are selected to represent them. Cartesian coordinates with Euler parameters are used to represent the overall large motion of the rigid bodies of the system [7] and of the flexible bodies' reference frames [3, 4, 8]. Different formulations for the description of flexible multibody systems have been proposed in the literature presenting relative advantages, and also drawbacks, with respect to the formulation used here [9-13]. However, the flexible multibody descriptions based on generalized elastic coordinates relative to body fixed frame not only use the standard linear finite element mass and stiffness matrices directly but also allow for the reduction of the number of elastic degrees of freedom, either by applying substructuring techniques [14], the Craig-Bampton method [3, 15], the mode superposition technique [8, 16-20] or other reduction techniques [21], leading to simpler and computationally efficient models.

One of the distinctive features of flexible multibody systems concerns the description of the relative motion between the system components using either kinematic joints or force elements such as contacting surfaces or lumped deformable elements. The body of kinematic joints developed is not only vast but also well reported in many textbooks for rigid multibody dynamics [7, 10]. More specialized kinematic joints, such as general spatial joint curves [22] or spatial cam joints [23], are defined among rigid bodies. The complete set of joints available in a general-purpose multibody code must include, for each particular type of joint, restrictions involving only rigid bodies or only flexible bodies or a flexible and a rigid body. Therefore, the effort put in the development and implementation of any joint is at 
least three times as much as the initial work done in the implementation of joints with rigid bodies only [24]. The concept of virtual bodies provides a general framework to develop general kinematic joints for flexible multibody systems with minimal effort, regardless of the flexible coordinates used. Initially, only a rigid constraint between the flexible and a massless rigid body is developed. Then, any kinematic joint that involve a flexible body is set with the massless rigid body instead, using the kinematic joint library involving only rigid bodies $[24,25]$. When using the virtual bodies the only type of joints that require the explicit use of generalized elastic coordinates are those that have deformable axis [26].

The flexible multibody involves, in general, beam and plate-like components that are made of composite materials. In order to use the material properties of composites a design variables for the optimal design it is necessary that the code in which the flexible multibody methodology is implemented includes the explicit formulation of the composite finite elements that can be used to model the system components. In this work, the description of composite beam elements follows the work proposed by Cesnik and Hodges [27] while the plate element is described in the work by Augusta Neto et al. [28]. The use of these finite elements in the framework of flexible multibody systems is described in different references and is not repeated here [30, 31].

The simplest and cheapest optimal problems are continuous local problems with a small number of design variables [32]. Global or integer optimization problems with a large number of variables are more complex and expensive to solve. Stochastic optimization algorithms, like simulated annealing methods or genetic algorithms, allow performing global optimization, generally requiring hundreds or even thousands of expensive simulation runs. Eberhard and co-workers used a stochastic evolution strategy in combination with parallel computing in order to reduce the computation times whilst keeping the inherent robustness [33]. Deterministic optimization algorithms have the tendency to reach local minima, not necessarily close to the global optimum [34]. When supported by efficient calculation of the system sensitivities they often converge rapidly towards a local minimum with smaller computation times. Due to the computational costs associated to the analysis of complex flexible multibody systems deterministic optimization algorithms are used in this work.

The simplest procedure to calculate the sensitivity derivatives is the finite-difference approximation [35]. However, small perturbations may result in errors in the derivative due to the limited accuracy of the dynamic response variation while large perturbations can lead to truncation errors [36]. Another drawback of this method concerns its poor numerical performance that results from requiring that additional analysis are performed for the perturbation of each additional design variables [38]. Analytical sensitivity formulations are alternatives for the exact evaluation of the derivatives of the dynamic response with respect to the design variables [39]. When the direct differentiation is used the integration of the dynamic and sensitivities equations are all done at the same time, thus the control of the time integration errors becomes more effective. The main disadvantage of the direct differentiation method is that it requires a large number of sensitivity equations to be derived analytically and implemented computationally, which involves cumbersome and prone to coding errors procedures. Recent developments in software technology have provided reliable automatic differentiation tools, such as automatic differentiation performs a transformation of functions, implemented computationally using Fortran 77, to obtain other functions also coded in the same language, which are the derivatives of the output of the first set of functions with respect to the design variables [40]. The use of automatic differentiation results in a new computer code, which provides fast and accurate gradients of the functions even for large and complex dynamic problems [41]. In order to use the advantages of the direct differentiation of the sensitivity equations and avoid the difficulties of its computer implementation, the 
automatic differentiation tool ADIFOR is used here. However, it is shown in this work that the code generated for the sensitivities cannot be used as a black box and, eventually, some parts of the code generated must be substituted by special purpose alternative algorithms.

The optimization of the multibody composite components is performed by taking the ply orientations of laminated as continuous design variables. The design of laminate materials in structural optimization is still an area of intensive research work, as shown in the review on structural optimization of composite structures by [42]. The multibody dynamic and sensitivities analysis code is linked with general optimization algorithms included in the package DOT/DOC [43]. The flexible slider-crank mechanism is used to present in detail the numerical and methodological issues that arise in the optimization of flexible multibody systems and a complex aerospace satellite antenna is used to present the application of the procedures to the design of a real life system.

\section{Flexible multibody systems analysis}

\subsection{Motion of a flexible body}

Let it be assumed that a flexible body is described in a coordinate system rigidly attached to a point on the flexible component, as depicted by Fig. 1. Let it also be assumed that the deformation of the flexible body is represented using the finite element method, with a condensed representation of the mass matrix, obtained by the diagonalization of the original consistent mass matrix [44]. The reference frame, located by vector $\mathbf{r}_{i}$ with respect to the inertia frame is fixed to the body center of mass [8]. The system equations of motion of this flexible body can be obtained by using Lagrange equations, as described by Gonçalves and Ambrósio [8], and therefore, not repeated here.

Using the principle of virtual powers, the Lagrange equations or other procedure, the equations of motion for a single flexible body are written as

$$
\mathbf{M}_{i} \ddot{\mathbf{q}}_{i}=\mathbf{g}_{i}+\mathbf{s}_{i}-\underline{\mathbf{K}}_{i} \mathbf{q}_{i}
$$

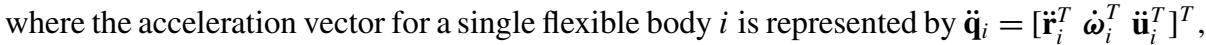
which contains the body fixed frame translation accelerations and the vector of the local nodal accelerations $\ddot{\mathbf{u}}_{i}^{\prime}=\left[\ddot{\boldsymbol{\delta}}^{\prime T} \ddot{\boldsymbol{\theta}}^{T}\right]_{i}^{T} . \ddot{\boldsymbol{\delta}}^{\prime}$ and $\dot{\boldsymbol{\theta}}^{\prime}$ are vectors of the nodal translations and angular accelerations, respectively, written with respect to the body fixed frame. The flexible

Fig. 1 Global position of node $k$ in the flexible body

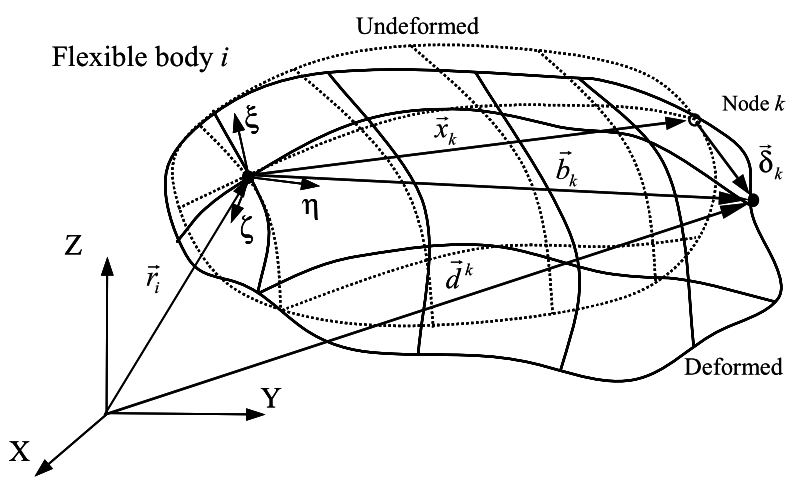


body mass matrix $\mathbf{M}_{i}$ is given by

$$
\mathbf{M}_{i}=\left[\begin{array}{cc|cc}
\sum \mathrm{m}_{k} \mathbf{I} & -\sum \mathrm{m}_{k} \mathbf{A}_{\mathbf{b}_{k}^{\prime}} & \sum \mathrm{m}_{k} \mathbf{A}_{k} \underline{\mathbf{I}}_{k}^{T} & \mathbf{0} \\
\sum \mathrm{m}_{k} \tilde{\mathbf{b}}_{k}^{\prime} \mathbf{A}^{T} & -\sum \mathrm{m}_{k} \tilde{\mathbf{b}}_{k}^{\prime} \tilde{\mathbf{b}}_{k}^{\prime}+\sum \mu_{k} \mathbf{I} & \sum \mathrm{m}_{k} \tilde{\mathbf{b}}_{k}^{\prime} \underline{\mathbf{I}}_{k}^{T} & \sum \mu_{k} \underline{\mathbf{I}}_{k}^{T} \\
\hline \sum \mathrm{m}_{k} \underline{\mathbf{I}}_{k} \mathbf{A}^{T} & -\sum \mathrm{m}_{k} \underline{\mathbf{I}}_{k} \tilde{\mathbf{b}}_{k}^{\prime} & \sum \mathrm{m}_{k} \underline{\mathbf{I}}_{k} \underline{\mathbf{I}}_{k}^{T} & \mathbf{0} \\
\mathbf{0} & \sum \mu_{k} \underline{\mathbf{I}}_{k} & \mathbf{0} & \sum \mu_{k} \underline{\mathbf{I}}_{k} \mathbf{I}_{k}^{T}
\end{array}\right]_{i}
$$

where $\mathbf{A}$ represents the transformation matrix from body $i$ fixed to inertial coordinates, $\mathbf{b}_{k}^{\prime}$ is the position of node $k$ of body $i$ with respect to the body fixed frame, $\mathrm{m}_{k}$ is the nodal mass, $\mu_{k}$ the nodal inertia, $\underline{\mathbf{I}}_{k}$ is a matrix filled with zeros except for the identity matrix associated to node $k$ and $(\tilde{\cdot})$ means the skew symmetric matrix associated to the quantity $(\cdot)$. In (1), $\mathbf{g}_{i}$ is the vector of external applied forces and $\mathbf{s}_{i}$ is the vector of gyroscopic forces of the flexible body $i$.

When a consistent mass matrix is used in the finite element description of the flexible body the inertia coupling terms, represented by the off-diagonal sub-matrices in (2), depend on the particular finite element shape functions used to formulate the finite elements. However, due to the diagonalization the structure of the mass matrix given by (2) becomes independent of the formulation used to describe the flexible body deformations and no special inertia-coupling coefficients have to be derived when using a particular finite element.

Finally, in (1) $\underline{\mathbf{K}}_{i}$ is an augmented stiffness matrix written as

$$
\underline{\mathbf{K}}_{i}=\left[\begin{array}{cc}
\mathbf{0} & \mathbf{0} \\
\mathbf{0} & \mathbf{K}_{f f}
\end{array}\right]_{i}
$$

where $\mathbf{K}_{f f}$ the standard finite element stiffness matrix of the flexible body $i$. Let it be assumed that the formulation is valid only for small linear elastic deformations, which implies that matrix $\mathbf{K}_{f f}$ is not dependent on the deformation of the flexible body. For more details on the derivation of (1) the reader is referred to Ref. [8].

\subsection{Flexible multibody equations of motion}

For a multibody system a set of kinematic constraints associated to the joints is defined as [8],

$$
\boldsymbol{\Phi}\left(\mathbf{q}_{r}, \mathbf{u}^{\prime}, t\right) \equiv \mathbf{0}
$$

where $\mathbf{q}_{r}$ are the coordinates associated to the rigid body motion and $t$ refers to the kinematic constraints that depend on time. Note that by using the virtual bodies to set the kinematic constraints the only equations that involve the generalized elastic coordinates are those associated to the rigid constraints between the flexible bodies and the virtual bodies [24, 25]. All other constraints only involve rigid bodies.

The constraints equations are added to the equilibrium equations of all bodies in the system, using Lagrange multipliers

$$
\mathbf{M} \ddot{\mathbf{q}}+\boldsymbol{\Phi}_{\mathbf{q}}^{T} \lambda=\mathbf{g}+\mathbf{s}-\underline{\mathbf{K}} \mathbf{q} .
$$

The second time derivatives of the constraint equations are necessary to support the solution of (5) and are represented as

$$
\ddot{\boldsymbol{\Phi}}(\ddot{\mathbf{q}}, \dot{\mathbf{q}}, \mathbf{q}, t) \equiv \boldsymbol{\Phi}_{\mathbf{q}} \ddot{\mathbf{q}}-\boldsymbol{\gamma}=\mathbf{0} .
$$


Therefore, the system of equations for the flexible multibody system that needs to be solved is

$$
\left[\begin{array}{ccc}
\mathbf{M}_{r} & \mathbf{M}_{r f} & \boldsymbol{\Phi}_{\mathbf{q}_{r}}^{T} \\
\mathbf{M}_{f r} & \mathbf{M}_{f f} & \boldsymbol{\Phi}_{\mathbf{q}_{f}}^{T} \\
\boldsymbol{\Phi}_{\mathbf{q}_{r}} & \boldsymbol{\Phi}_{\mathbf{q}_{f}} & \mathbf{0}
\end{array}\right]\left\{\begin{array}{c}
\ddot{\mathbf{q}}_{r} \\
\ddot{\mathbf{u}}^{\prime} \\
\boldsymbol{\lambda}
\end{array}\right\}=\left\{\begin{array}{c}
\mathbf{g}_{r} \\
\mathbf{g}_{f} \\
\boldsymbol{\gamma}
\end{array}\right\}-\left\{\begin{array}{c}
\mathbf{s}_{r} \\
\mathbf{s}_{f} \\
\mathbf{0}
\end{array}\right\}-\left\{\begin{array}{c}
\mathbf{0} \\
\mathbf{K}_{f f} \mathbf{u}^{\prime} \\
\mathbf{0}
\end{array}\right\} .
$$

The Jacobian matrix $\boldsymbol{\Phi}_{\mathbf{q}}^{T}$ and the right-hand side vector $\boldsymbol{\gamma}$ depend on the type of kinematic constraints used. Due to the sparsity of the leading matrix of the left-hand side of (7), a sparse matrix solver is employed to solve the system of equations [19, 45].

The component mode synthesis the flexible body is used to reduce the number of generalized elastic coordinates required to describe the flexible multibody model. Consequently

$$
\mathbf{u}^{\prime}=\mathbf{X} \mathbf{w}
$$

where vector $\mathbf{w}$ represents the contributions of the vibration modes towards the nodal displacements and $\mathbf{X}$ is the modal matrix. Due to the reference conditions, the modes of vibration used here are constrained modes and due to the assumption of linear elastic deformations the modal matrix is invariant. The reduced equations of motion for the flexible body are [8]

$$
\left[\begin{array}{ccc}
\mathbf{M}_{r} & \mathbf{M}_{r f} \mathbf{X} & \boldsymbol{\Phi}_{\mathbf{q}_{r}}^{T} \\
\mathbf{X}^{T} \mathbf{M}_{f r} & \mathbf{I} & \mathbf{X}^{T} \boldsymbol{\Phi}_{\mathbf{q}_{f}}^{T} \\
\boldsymbol{\Phi}_{\mathbf{q}_{r}} & \boldsymbol{\Phi}_{\mathbf{q}_{f}} \mathbf{X} & \mathbf{0}
\end{array}\right]\left\{\begin{array}{c}
\ddot{\mathbf{q}}_{r} \\
\ddot{\mathbf{w}} \\
\boldsymbol{\lambda}
\end{array}\right\}=\left\{\begin{array}{c}
\mathbf{g}_{r} \\
\mathbf{X}^{T} \mathbf{g}_{f} \\
\boldsymbol{\gamma}
\end{array}\right\}-\left\{\begin{array}{c}
\mathbf{s}_{r} \\
\mathbf{X}^{T} \mathbf{s}_{f} \\
\mathbf{0}
\end{array}\right\}-\left\{\begin{array}{c}
\mathbf{0} \\
\boldsymbol{\Lambda} \mathbf{w} \\
\mathbf{0}
\end{array}\right\}
$$

where $\boldsymbol{\Lambda}$ is a diagonal matrix that has the squares of the natural frequencies associated to the modes of vibration selected. It must be noticed that the choice of modes is an important matter that conditions accuracy and efficiency. For a more detailed discussion on the selection of the modes used the interested reader is referred to Refs. [15-20, 46].

\subsection{Composite materials finite elements}

In this work, the composite finite element used for the study of laminated plates is based on the Mindlin-Reissner plate theory. At the element level and in local coordinates, the element stiffness matrix is written as [29,47]

$$
K_{f f}^{(e)}=\int_{0}^{1} \int_{0}^{1-\eta}\left[\begin{array}{ccc}
\mathbf{B}_{m}^{T} \mathbf{D}_{m} \mathbf{B}_{m} & \mathbf{B}_{m}^{T} \mathbf{D}_{m b} \mathbf{B}_{b} & \mathbf{0} \\
\mathbf{B}_{b}^{T} \mathbf{D}_{b m} \mathbf{B}_{m} & \mathbf{B}_{b}^{T} \mathbf{D}_{b} \mathbf{B}_{b} & \mathbf{0} \\
\mathbf{0} & \mathbf{0} & \mathbf{B}_{s}^{T} \mathbf{D}_{s} \mathbf{B}_{s}
\end{array}\right]^{(e)}|\mathbf{J}| d \xi d \eta .
$$

The strain-displacement matrix is denoted by $\mathbf{B}$ while $\mathbf{D}$ is the elasticity matrix and $|\mathbf{J}|$ is the determinant of the Jacobian matrix. The subscripts $m, b$ and $s$ stand for membrane, bending and shear, respectively. Because each layer may have different properties, the elasticity matrix $\mathbf{D}$ is evaluated as a summations carried out over the thickness of all the layers. Therefore, equivalent single layer theories produce equivalent stiffness matrices as weighted averages of the individual layer stiffness through the thickness. These matrices are depen- 

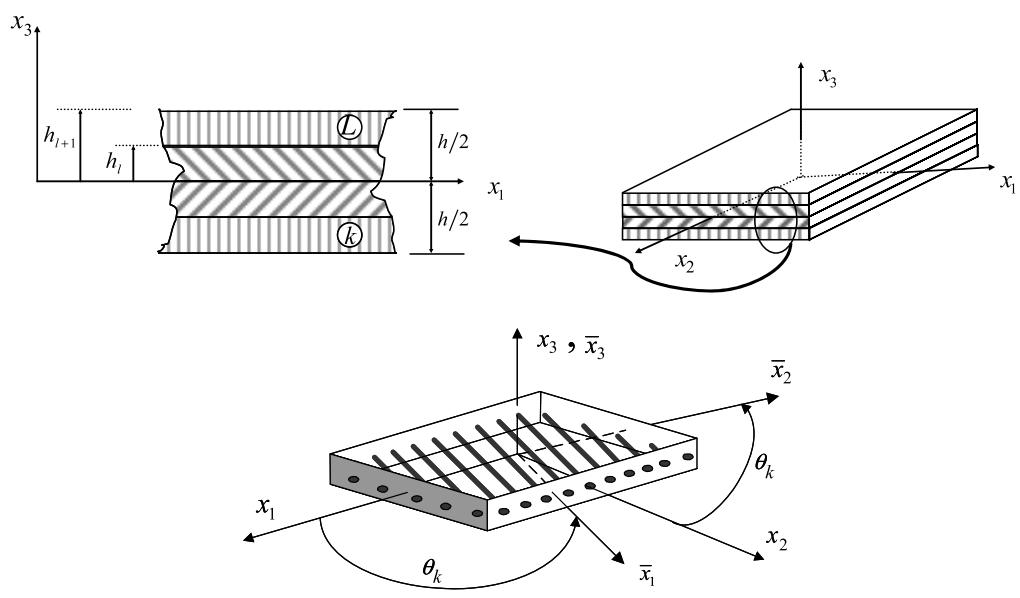

Fig. 2 Coordinate system and layer numbering used for a typical laminated plate and orthotropic layer with the principal material directions

dent of each layer orientation and given by

$$
\begin{aligned}
\left(\mathbf{D}_{m}, \mathbf{D}_{b}, \mathbf{D}_{m b}, \mathbf{D}_{s}\right) & =\sum_{k=1}^{n}\left(\mathbf{D}_{m}, \mathbf{D}_{b}, \mathbf{D}_{m b}, \mathbf{D}_{s}\right)_{k} \\
& =\sum_{k=1}^{n}\left(\mathbf{C}_{3 x 3}^{1} H_{1}, \mathbf{C}_{3 x 3}^{1} H_{2}, \mathbf{C}_{3 x 3}^{1} H_{3}, \mathbf{C}_{2 x 2}^{2} H\right)_{k}
\end{aligned}
$$

with,

$$
H_{n}=\int_{h_{l-1}}^{h_{l}}\left(x_{3}^{n-1}\right) d z=\frac{1}{n}\left(h_{l+1}^{n}-h_{l}^{n}\right)
$$

where $h_{i}$ is defined in Fig. 2. At element level, in local coordinates, the mass matrix is

$$
\mathbf{M}_{f f}^{(e)}=\int_{0}^{1} \int_{0}^{1-\eta} \rho^{(e)}\left(\mathbf{S}^{T} \mathbf{m} \mathbf{S}\right)^{(e)}|\mathbf{J}| d \xi d \eta
$$

where $\mathbf{m}$ is a matrix that contains the inertial terms and $\rho$ represents the specific mass of the element. The diagonalization of the mass matrix is used in what follows [44].

In order to do the analysis of composite plates, a triangular finite element is developed. The finite element is based in the theory described and has six degrees of freedom per node, i.e., $u_{1}^{0}, u_{2}^{0}, u_{3}^{0}, \phi_{1}, \phi_{2}$ and $\phi_{3}$. A detailed description of this composite plate element is given in Ref. [47].

The composite beam element available for flexible multibody models is that proposed by Cesnik and Hodges [48] where the cross-sections properties are found using an asymptotic procedure that involves a two-dimensional finite element analysis over the beam crosssection. The elasticity matrix for the beam is obtained by using the code VABS, which is a standard 2-D finite element code for arbitrary materials and geometries [49]. The source code for the composite beam element, as well as for the composite plate element, is available to be used for the automatic differentiation. A detailed description of this composite beam element can be obtained in Refs. [49, 50]. 


\section{Optimization of Multibody Systems}

The general optimization problem is stated in a mathematically form as the problem of finding the minimum of an objective function for a given vector $\mathbf{b}$ of design variables that are subject to specific constraints. This is,

$$
\begin{array}{lll}
\text { minimize } & \Psi_{0}(\mathbf{b}) & \text { (objective function), } \\
\text { subject to } & \boldsymbol{\Psi}_{i}(\mathbf{b}) \leq 0, \quad i=1, \ldots, p & \text { (inequality constraints), } \\
& \boldsymbol{\Psi}_{i}(\mathbf{b})=0, \quad i=p+1, \ldots, p+m & \text { (equality constraints), } \\
& \mathbf{b}_{L} \leq(\mathbf{b}) \leq \mathbf{b}_{U} & \text { (side constraints) }
\end{array}
$$

where the indices $U_{i}$ and $L_{i}$ refer to the upper and lower limit constraints of the design variables. These upper and lower limits result from technologic and/or physical requirements.

\subsection{Optimization criteria}

Different optimization problems in multibody systems require, in general, different criteria and design constraints. In multibody systems all terms of the equations of motion may be functions of the design parameters. The objective function is expressed by [51]

$$
\Psi_{0}=\Psi_{0}(\mathbf{b}, \mathbf{z}, \lambda, t)
$$

where the vector of the state variables $\mathbf{z}$ includes the system positions, velocities and accelerations. The variables of the state vector may depend on time and on the design variables as

$$
\mathbf{z}(\mathbf{b}, t)=(\mathbf{q}(\mathbf{b}, t), \dot{\mathbf{q}}(\mathbf{b}, t), \ddot{\mathbf{q}}(\mathbf{b}, t)) .
$$

Note also that the most common objective functions, in multibody problems, deal with a maximum value or with the integral of a given quantity. The objectives for the flexible multibody systems designs concern the stiffening of the flexible components. Therefore, the objective functions considered here are measures of the structural deformations.

\subsection{Minimization of the maximum deformation energy}

The elastic deformation energy is used here as a global measure of deformation. For an instant of time $t$, the elastic deformation energy of a body $i$ is

$$
U_{i}\left(\mathbf{u}_{i}^{\prime}, t\right)=1 / 2 \mathbf{u}_{i}^{\prime T} \mathbf{K}_{f f_{i}} \mathbf{u}_{i}^{\prime} .
$$

A mini-max optimization problem, for the time interval between $t_{0}$ and $t_{e}$ is defined to minimize the maximum elastic deformation energy of body $i$. This optimization problem is

$$
\operatorname{minimize} \quad \Psi_{0}^{\max }=\max U_{i}\left(\mathbf{u}_{i}^{\prime}, t\right), \quad \text { for } t_{0} \leq t \leq t_{e}
$$

where $t_{0} \leq t \leq t_{e}$ is the time interval in which the minimum is searched. However, the instant, for which the function has its maximum value may vary during the optimization process. One form of dealing with this problem is to introduce an extra design variable, $b_{n+1}$ and make the objective function equal to the value of such variable $[37,52]$. The new optimization problem is defined as

$$
\text { minimize } \Psi_{0}=b_{n+1}
$$


with the additional time-dependent constraint

$$
\Psi_{n+1}=U_{i}\left(\mathbf{u}_{i}^{\prime}, t\right)-b_{n+1} \leq 0, \quad \text { for } t_{0} \leq t \leq t_{e}
$$

this additional constraint ensures that the dynamic response is below the maximum value defined by auxiliary variable $b_{n+1}$. This approach poses some difficulties for the search direction calculus, and can lead to small steps in the line search method, or even to the stall of the optimization process. To overcome these difficulties, the max value point is handled directly only in the optimization process [52].

\subsection{Minimization of an integral type criteria}

The integral type objective function may be used to represent mean values of the response along time, accumulated values or other special criteria. This type of objective functions is common in vehicle design, in which comfort or injury criteria are defined in this manner and often are used in their optimization process [53]. The optimal problem can be expressed here as the minimization of the integral of the deformation energy in the analysis time period

$$
\Psi_{0}=\int_{t_{0}}^{t_{e}} U_{i}\left(\mathbf{u}_{i}^{\prime}, t\right) d t
$$

Note that the use of this type of functions generally requires that a constraint on the maximum value of the quantity being integrated is also defined.

\subsection{Time-dependent constraint}

Mathematical programming algorithms generally cannot deal with parametric constraints that are time dependent, such as

$$
\Psi_{i}=f_{i}(\mathbf{b}, \mathbf{z}(t), \lambda(t), t) \leq c, \quad t_{i} \leq t \leq t_{e}
$$

or even with constraints such as the one described by (20). Such constraints have to be reformulated to remove their time dependency. The most straightforward way to remove the time dependency of the original constraint is to discretize the time interval into time points. Then, the original constraint represented by (27) is replaced by $n_{t p}$ constraints written as [54]

$$
\Psi_{i}=f_{i}\left(\mathbf{b}, \mathbf{z}\left(t_{k}\right), \lambda\left(t_{k}\right), t_{k}\right) \leq c, \quad k=1, \ldots, n_{t p} .
$$

The distribution of the time points has to be sufficiently dense to avoid large constraints violations between two adjacent time points [55]. However, discretizing time dependent constraints can increase greatly the number of constraints, and thereby the cost of optimization [37]. A balance can be achieved by using a discretization that involves only critical points related with the existence of local maximums or minimums of the function [56].

\subsection{Optimization algorithms}

In dynamic problems, the evaluation of the system dynamic behavior requires the numerical integration of the equation of motion. The time dependency of this system makes these optimization problems more complex and requires that special techniques must be used in the solution process. Both deterministic and stochastic optimization methods can be applied. 
Eberhard et al. [33] and He and McPhee [57] have successfully used a stochastic evolution strategy in a parallel computing environment to reduce computation time. However, when a single processor computer is used the simultaneous analysis of different systems is not possible and, consequently, some of the advantages of stochastic optimization methodologies disappear. In this work, a deterministic optimization method is used, the modified method of feasible directions, as implemented in DOT [43].

\section{Sensitivities of the flexible multibody system}

The optimization process requires the evaluation of the gradients of the objective functions, which must be supplied to the optimization algorithm. These gradients, or sensitivities, can be obtained by finite differences or derived analytically and supplied as functions called by the computer code that controls the optimization. To obtain the sensitivities let a typical objective function be defined as

$$
\psi_{0}=f_{0}(\mathbf{b}, \mathbf{z}, \lambda, t)
$$

where the vector of state variables $\mathbf{z}$ denotes the positions, velocities and accelerations that are dependent on time and on the design parameters, as

$$
\mathbf{z}(\mathbf{b}, t)=\mathbf{z}(\mathbf{q}(\mathbf{b}, t), \dot{\mathbf{q}}(\mathbf{b}, t), \ddot{\mathbf{q}}(\mathbf{b}, t)) .
$$

The solution of the optimal problem, by the methods used in this work, requires the computation of the derivative of all objective and constraint functions with respect to the design variables. Such gradients are written as

$$
\nabla \boldsymbol{\psi}_{i}=\frac{d \boldsymbol{\psi}_{i}}{d \mathbf{b}}, \quad i=0, \ldots, n
$$

the derivatives are calculated by using the chain rule, as

$$
\frac{d \psi_{i}}{d \mathbf{b}}=\frac{\partial \psi_{i}}{\partial \mathbf{b}}+\frac{\partial \psi_{i}}{\partial \mathbf{q}} \frac{d \mathbf{q}}{d \mathbf{b}}+\frac{\partial \psi_{i}}{\partial \dot{\mathbf{q}}} \frac{d \dot{\mathbf{q}}}{d \mathbf{b}}+\frac{\partial \psi_{i}}{\partial \ddot{\mathbf{q}}} \frac{d \ddot{\mathbf{q}}}{d \mathbf{b}}+\frac{\partial \psi_{i}}{\partial \lambda} \frac{d \lambda}{d \mathbf{b}}
$$

In a more compact notation, the partial derivatives of the state variables with respect to the design variables, are represented as

$$
\frac{d \psi_{i}}{d \mathbf{b}}=\frac{\partial \psi_{i}}{\partial \mathbf{b}}+\frac{\partial \psi_{i}}{\partial \mathbf{q}} \mathbf{q}_{\mathbf{b}}+\frac{\partial \psi_{i}}{\partial \dot{\mathbf{q}}} \dot{\mathbf{q}}_{\mathbf{b}}+\frac{\partial \psi_{i}}{\partial \ddot{\mathbf{q}}} \ddot{\mathbf{q}}_{\mathbf{b}}+\frac{\partial \psi_{i}}{\partial \lambda} \lambda_{\mathbf{b}}
$$

In evaluating (24), the computations associated with the terms $\partial \psi_{i} / \partial \mathbf{b}, \partial \psi_{i} / \partial \mathbf{q}, \partial \psi_{i} / \partial \dot{\mathbf{q}}$, $\partial \psi_{i} / \partial \ddot{\mathbf{q}}$ and $\partial \psi_{i} / \partial \lambda$ are simple due to the fact that the design variables and the state variables are explicit. The computation of the sensitivities of the state variables with respect the design variables is rather complex, requiring a large computational effort. These derivatives can be obtained by numerical or by analytical methods.

\subsection{Finite difference method}

The finite-difference method is very often used as a technique to approximate the sensitivities [35-37]. This method, based in the Taylor series expansion of a function $f$, for 
a perturbation $\Delta \mathbf{b}$ of the design parameters vector $\mathbf{b}$, is expressed as

$$
\nabla f(\mathbf{b})=\frac{f(\mathbf{b}+1 / 2 \Delta \mathbf{b})-f(\mathbf{b}-1 / 2 \Delta \mathbf{b})}{\Delta \mathbf{b}}
$$

where the expression is the central finite difference. In applications to multibody dynamics, the use of finite differences is an attractive approach due to its simplicity and simple implementation. However, the accuracy of this method is lower than the analytical method. The major drawback of this method is the high number of functions evaluations required. For instance, the use of (29) requires for each design variable two new dynamic analysis to evaluate the perturbed response.

In applying finite differences, the selection of difference step size $\Delta \mathbf{b}$ is also a concern. The selection of a large step size leads to errors in the derivatives due to truncation of the operator. The selection of a small step size can lead to condition errors in the derivative due to the limited floating point precision of the computer or to the inaccurate calculation of function $f(\mathbf{b}+\Delta \mathbf{b})$ in particular points. The time integration used in the dynamic response optimization tends to aggravate the loss in accuracy of the finite differences. This approach is used here, mostly, as a measure for the correct implementation of analytical sensitivities.

\subsection{Direct differentiation of the equations of motion}

For a rigid and flexible multibody system the equations of motion are given by (9). To calculate the sensitivities, this equation is differentiated with respect to the design variables $\mathbf{b}$, leading to

$$
\left[\begin{array}{ccc}
\mathbf{M}_{r r} & \overline{\mathbf{M}}_{r f} & \boldsymbol{\Phi}_{\mathbf{q}_{r}}^{T} \\
\overline{\mathbf{M}}_{f r} & \overline{\mathbf{M}}_{f f} & \boldsymbol{\Phi}_{\mathbf{w}}^{T} \\
\boldsymbol{\Phi}_{\mathbf{q}_{r}} & \boldsymbol{\Phi}_{\mathbf{w}} & \mathbf{0}
\end{array}\right]\left[\begin{array}{c}
\ddot{\mathbf{q}}_{r_{\mathbf{b}}} \\
\ddot{\mathbf{w}}_{\mathbf{b}} \\
\boldsymbol{\lambda}_{\mathbf{b}}
\end{array}\right]=\left[\begin{array}{c}
\overline{\mathbf{Q}}_{\mathbf{b}}^{*} \\
\overline{\mathbf{R}}_{\mathbf{b}}^{*} \\
\overline{\boldsymbol{\gamma}}_{\mathbf{b}}^{*}
\end{array}\right]
$$

where the notation used relates with that in (9) by defining $\overline{\mathbf{M}}_{f f}=\mathbf{X}^{T} \mathbf{M}_{f f} \mathbf{X}, \overline{\mathbf{M}}_{r f}=$ $\mathbf{M}_{r f} \mathbf{X}, \overline{\mathbf{M}}_{f r}=\mathbf{X}^{T} \mathbf{M}_{f r}, \boldsymbol{\Phi}_{\mathbf{w}}=\boldsymbol{\Phi}_{\mathbf{q}_{f}} \mathbf{X}$ and $\boldsymbol{\Phi}_{\mathbf{w}}^{T}=\mathbf{X}^{T} \boldsymbol{\Phi}_{\mathbf{q}_{f}}^{T}$. In (30) the quantities in the right-hand side vector are given by

$$
\begin{aligned}
\overline{\mathbf{Q}}_{\mathbf{b}}^{*}= & \frac{\partial}{\partial \mathbf{q}_{r}}\left(\mathbf{g}_{r}-\mathbf{s}_{r}-\mathbf{M}_{r r} \hat{\ddot{\mathbf{q}}}_{r}-\overline{\mathbf{M}}_{r f} \hat{\ddot{\mathbf{w}}}-\boldsymbol{\Phi}_{\mathbf{q}_{r}}^{T} \hat{\lambda}\right) \mathbf{q}_{r_{\mathbf{b}}}+\frac{\partial}{\partial \dot{\mathbf{q}}_{r}}\left(\mathbf{g}_{r}-\mathbf{s}_{r}\right) \dot{\mathbf{q}}_{r_{\mathbf{b}}} \\
& +\frac{\partial}{\partial \mathbf{w}}\left(\mathbf{g}_{r}-\mathbf{s}_{r}-\mathbf{M}_{r r} \hat{\ddot{\mathbf{q}}}_{r}-\overline{\mathbf{M}}_{r f} \hat{\ddot{\mathbf{w}}}-\boldsymbol{\Phi}_{\mathbf{q}_{r}}^{T} \hat{\lambda}\right) \mathbf{w}_{\mathbf{b}}+\frac{\partial}{\partial \dot{\mathbf{w}}}\left(\mathbf{g}_{r}-\mathbf{s}_{r}\right) \dot{\mathbf{w}}_{\mathbf{b}} \\
& +\frac{\partial}{\partial \mathbf{b}}\left(\mathbf{g}_{r}-\mathbf{s}_{r}-\mathbf{M}_{r r} \hat{\hat{\mathbf{q}}}_{r}-\overline{\mathbf{M}}_{r f} \hat{\hat{\mathbf{w}}}-\boldsymbol{\Phi}_{\mathbf{q}_{r}}^{T} \hat{\lambda}\right), \\
\overline{\mathbf{R}}_{\mathbf{b}}^{*}= & \frac{\partial}{\partial \mathbf{q}_{r}}\left(\overline{\mathbf{g}}_{f}-\overline{\mathbf{s}}_{f}-\overline{\mathbf{K}}_{f f} \mathbf{w}-\overline{\mathbf{C}}_{f f} \dot{\mathbf{w}}-\overline{\mathbf{M}}_{f r} \hat{\mathbf{q}}_{r}-\overline{\mathbf{M}}_{f f} \hat{\ddot{\mathbf{w}}}-\boldsymbol{\Phi}_{\mathbf{w}}^{T} \hat{\lambda}\right) \mathbf{q}_{r_{\mathbf{b}}} \\
& +\frac{\partial}{\partial \mathbf{w}}\left(\overline{\mathbf{g}}_{f}-\overline{\mathbf{s}}_{f}-\overline{\mathbf{K}}_{f f} \mathbf{w}-\overline{\mathbf{C}}_{f f} \dot{\mathbf{w}}-\overline{\mathbf{M}}_{f r} \hat{\ddot{\mathbf{q}}}_{r}-\overline{\mathbf{M}}_{f f} \hat{\ddot{\mathbf{w}}}-\boldsymbol{\Phi}_{\mathbf{w}}^{T} \hat{\lambda}\right) \mathbf{w}_{\mathbf{b}} \\
& +\frac{\partial}{\partial \mathbf{b}}\left(\overline{\mathbf{g}}_{f}-\overline{\mathbf{s}}_{f}-\overline{\mathbf{K}}_{f f} \mathbf{w}-\overline{\mathbf{C}}_{f f} \dot{\mathbf{w}}-\overline{\mathbf{M}}_{f r} \hat{\hat{\mathbf{q}}}_{r}-\overline{\mathbf{M}}_{f f} \hat{\hat{\mathbf{w}}}-\boldsymbol{\Phi}_{\mathbf{w}}^{T} \hat{\lambda}\right), \\
& +\frac{\partial}{\partial \dot{\mathbf{q}}_{r}}\left(\overline{\mathbf{g}}_{f}-\overline{\mathbf{s}}_{f}\right) \dot{\mathbf{q}}_{r_{\mathbf{b}}}+\frac{\partial}{\partial \dot{\mathbf{w}}}\left(\overline{\mathbf{g}}_{f}-\overline{\mathbf{s}}_{f}-\overline{\mathbf{C}}_{f f} \dot{\mathbf{w}}\right) \dot{\mathbf{w}}_{\mathbf{b}},
\end{aligned}
$$




$$
\begin{aligned}
\overline{\boldsymbol{\gamma}}_{\mathbf{b}}^{*}= & \frac{\partial}{\partial \mathbf{q}_{r}}\left(\overline{\boldsymbol{\gamma}}-\boldsymbol{\Phi}_{\mathbf{q}_{r}} \hat{\hat{\mathbf{q}}}_{r}-\boldsymbol{\Phi}_{\mathbf{w}} \hat{\hat{\mathbf{w}}}\right) \mathbf{q}_{r_{\mathbf{b}}}+\frac{\partial}{\partial \mathbf{w}}\left(\overline{\boldsymbol{\gamma}}-\boldsymbol{\Phi}_{\mathbf{q}_{r}} \hat{\hat{\mathbf{q}}}_{r}-\boldsymbol{\Phi}_{\mathbf{w}} \hat{\hat{\mathbf{w}}}\right) \mathbf{w}_{\mathbf{b}} \\
& +\frac{\partial}{\partial \mathbf{b}}\left(\bar{\gamma}-\boldsymbol{\Phi}_{\mathbf{q}_{r}} \hat{\mathbf{q}}_{r}-\boldsymbol{\Phi}_{\mathbf{w}} \hat{\hat{\mathbf{w}}}\right)+\frac{\partial \bar{\gamma}}{\partial \dot{\mathbf{q}}_{r}} \dot{\mathbf{q}}_{r_{\mathbf{b}}}+\frac{\partial \bar{\gamma}}{\partial \dot{\mathbf{w}}} \dot{\mathbf{w}}_{\mathbf{b}}
\end{aligned}
$$

where the overscript $\hat{\imath}$ denotes that the quantity $\cdot$ remains constant during the differentiation process.

After the solution of (30), i.e., after solving the linear system of equations to obtain the sensitivities, $\ddot{\mathbf{q}}_{r_{\mathbf{b}}}, \ddot{\mathbf{w}}_{\mathbf{b}}$ and $\lambda_{\mathbf{b}}$ the sensitivities of the positions and of the velocities are obtained by direct integration of $\dot{\mathbf{q}}_{r_{\mathbf{b}}}, \dot{\mathbf{w}}_{\mathbf{b}}, \ddot{\mathbf{q}}_{r_{\mathbf{b}}}, \ddot{\mathbf{w}}_{\mathbf{b}}$. The numerical process is started by providing the initial conditions of the sensitivities, calculated using the analytic sensitivities derived with the automatic differentiation tool. Another alternative for providing the initial sensitivities and their time derivatives is the use of the numerical sensitivities expressed by (29) for the initial conditions of the system.

The analytical derivation of the partial derivatives in (31), (32) and (33) is generally difficult for complex systems, as in the case of flexible multibody systems. The evaluation of $\overline{\mathbf{Q}}_{\mathbf{b}}^{*}, \overline{\mathbf{R}}_{\mathbf{b}}^{*}$ and $\overline{\boldsymbol{\gamma}}_{\mathbf{b}}^{*}$, in particular, requires further considerations not only due to the need to evaluate the sensitivities of the different matrices and vectors but also because the modal matrix sensitivities calculations pose extra challenges. Consequently, a cumbersome and error-prone to coding effort may be required. The use of automatic differentiation tool, ADIFOR [40] helps circumventing this difficulty, provided that the dynamic analysis code is available, in FORTRAN, and structured in a convenient way.

A weakness of automatic differentiation tools concerns the reliability of the differential algorithm used for the solution of the eigenproblem, which is required when the mode component synthesis is used in the multibody formulation [58]. Therefore, a close look on the use of the results of the eigenproblem in the framework of flexible multibody systems is taken in this work, with the objective of devising a strategy that overcomes the deficiency of the differentiated code used to calculate the sensitivities of the modes of vibration and natural frequencies.

Due to the coordinate reduction, which uses the component mode synthesis, the nodal displacements of the flexible body are described by (8). The sensitivity of the nodal displacement vector is obtained by computing the derivative of (8) with respect the design variables, as

$$
\frac{d \mathbf{u}^{\prime}}{d \mathbf{b}}=\frac{\partial \mathbf{X}}{\partial \mathbf{b}} \mathbf{w}+\mathbf{X} \frac{\partial \mathbf{w}}{\partial \mathbf{b}}=\mathbf{X}_{\mathbf{b}} \mathbf{w}+\mathbf{X w}_{\mathbf{b}}
$$

where $\mathbf{X}_{\mathbf{b}}$ are the sensitivities of the eigenmodes to the design variables. Equation (34) relates the modal sensitivities with the nodal sensitivities. Haftka and Gürdal [37], suggest two different approaches to evaluate this transformation: the fixed-mode approach, where the derivatives of vibration modes are neglected; the updated-mode approach, where the derivatives of vibration modes are retained. The modal stiffness matrix derivative using the updated-mode approach is computed as

$$
\frac{\partial}{\partial \mathbf{b}}\left(\mathbf{X}^{T} \mathbf{K}_{f f} \mathbf{X}\right)=\frac{\partial \mathbf{X}^{T}}{\partial \mathbf{b}} \mathbf{K}_{f f} \mathbf{X}+\mathbf{X}^{T} \frac{\partial \mathbf{K}_{f f}}{\partial \mathbf{b}} \mathbf{X}+\mathbf{X}^{T} \mathbf{K}_{f f} \frac{\partial \mathbf{X}}{\partial \mathbf{b}}
$$

When using the fixed-mode approach the sensitivity of the modal stiffness matrix is

$$
\frac{\partial}{\partial \mathbf{b}}\left(\mathbf{X}^{T} \mathbf{K}_{f f} \mathbf{X}\right)=\mathbf{X}^{T} \frac{\partial \mathbf{K}_{f f}}{\partial \mathbf{b}} \mathbf{X}
$$


It should be noted that although automatic differentiation is used to calculate the modal stiffness sensitivities, the problems that arise from having repeated eigenvalues are similar to those observed when using direct implementations of analytic sensitivities. The computation of the sensitivities of the eigenmodes can be evaluated using the Nelson scheme [59] for the case of distinct eigenvalues. However, when repeated eigenvalues occur, Ojavo's method is used [60]. These methods are implemented and used in this work instead of the differentiated part of the code that would 'solve' the eigenproblem.

\section{Demonstrative example}

A spatial slider-crank mechanism with a flexible connecting rod, modeled with composite plates, is used to demonstrate the features of the methodology proposed. The objective is to find the ply orientations of the plate laminate stacking sequence which is best suited to the dynamic load conditions. The elastic energy of the flexible rod, the maximum deformation energy and local torsion measures are used as potential objective functions for the rod design.

\subsection{Slider-crank mechanism with flexible connecting rod}

A slider-crank with the dimensions shown in Fig. 3 includes a connecting rod constituted by a composite plate with a thickness of $0.0075 \mathrm{~m}$ and a depth of $0.04 \mathrm{~m}$ being its body fixed coordinate frame attached to the plate middle surface, and its axis $\xi \eta$ parallel to the surface. The composite material properties are $E_{1}=128 \mathrm{GPa}, E_{2}=E_{3}=10.1 \mathrm{GPa}, G_{12}=G_{13}=$ $G_{23}=5.36 \mathrm{GPa}, v=0.23, \rho=1586 \mathrm{~kg} / \mathrm{m}^{3}$. The crank has a constant angular velocity of $124.8 \mathrm{rad} / \mathrm{s}$. Three models of laminated plates, with one, two and three layers are used as study cases.

Four different lay-ups, i.e., fiber orientations of a layer, described in Table 1, are considered in the plate models for the sensitivity analysis. In this case, only a single layer is considered in each plate model. The objective of the sensitivity analysis is to show the performance of the different procedures used to calculate the sensitivities of flexible multibody systems made of composite materials.

The analytic sensitivities of the normalized displacement of the mid node of the plate are plotted for lay-ups 1 and 7 in Figs. 4 and 5, respectively, using the fixed and the updated mode approaches. The results emphasize that although for some lay-ups, the updated and fixed mode approaches lead to the same results, such as for lay-up 7, in other cases, a major difference between the sensitivities obtained by these methods may exist. Therefore, it is not recommended in the use of fixed mode approach in the framework of the optimization of flexible multibody systems with composite materials.

Fig. 3 Slider-crank mechanisms with a composite plate

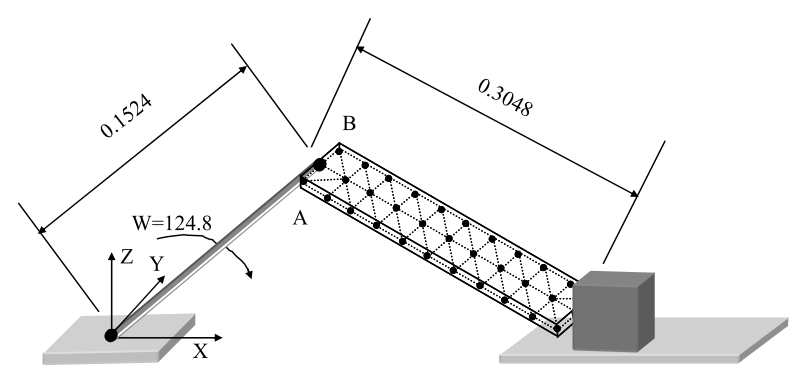


Table 1 Fiber orientations of the composite material in the four lay-ups of the composite plates used

Fig. 4 Sensitivity of the connecting rod mid-node displacement using lay-up 1

Fig. 5 Sensitivity of the connecting rod mid-node displacement for lay-up 7

\begin{tabular}{lllll}
\hline Lay-ups & 1 & 2 & 6 & 7 \\
\hline Layer orientation & $0^{\circ}$ & $15^{\circ}$ & $75^{\circ}$ & $90^{\circ}$ \\
\hline
\end{tabular}
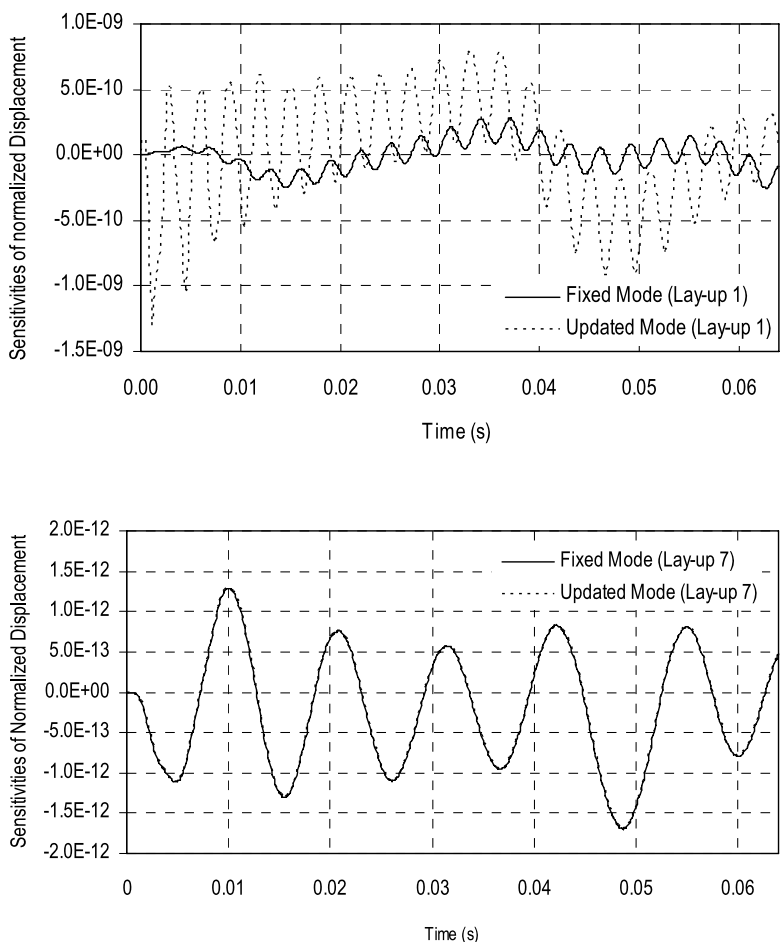

In Figs. 6 and 7, the analytic-numerical and numerical sensitivities, computed by the update-mode approach and the forward difference method with several finite difference step sizes, are presented for lay-ups 2 and 6, respectively.

Figures 6 and 7 show that the selection of the finite difference variation influences the sensitivities obtained using the forward difference method. The sensitivities obtained by the analytic and numerical methods are dependent on the lay-up considered and on the finite difference variation. A relation between perturbation size and the accuracy of the finite difference sensitivity is not always observed. For all these reasons, it is not recommended in the use of finite differences in optimization of flexible multibody systems made of composite materials.

\subsection{Rod deformation optimization - integral of the deformation energy}

The objective function represented by (21) is used here for the optimization of the material of the connecting rod of the slider crank presented in Fig. 2. An extra constraint that limits maximum value of the deformation energy not to exceed the maximum deformation energy for the initial design, designated by $c$, is defined as

$$
\Psi_{1}(\boldsymbol{\theta})=f_{0} \leq c .
$$


Fig. 6 Analytical and numerical sensitivities of the mid-node displacement with lay-up 2

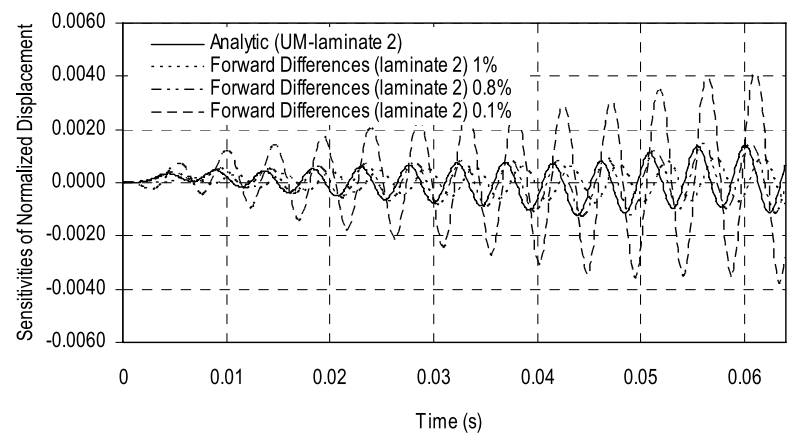

Fig. 7 Analytical and numerical sensitivities of the connecting rod mid-node displacement for lay-up 6

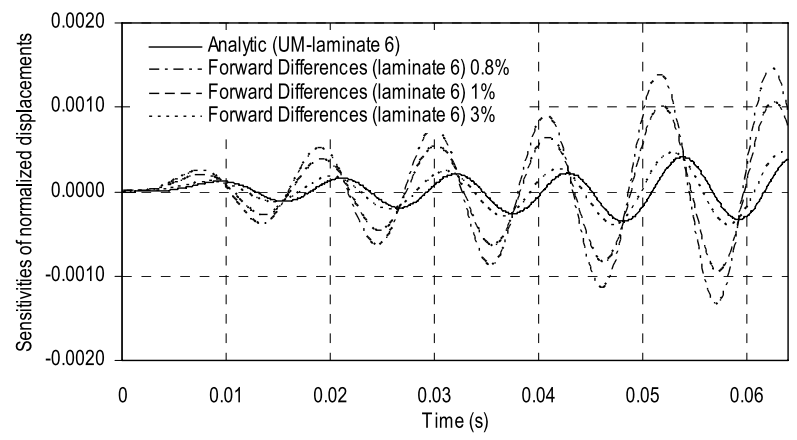

Table 2 Optimization design variables and boundary values

\begin{tabular}{lllllll}
\hline Laminate & $\begin{array}{l}\text { Layer } \\
\text { thicknesses } \\
(\mathrm{mm})\end{array}$ & $\begin{array}{l}\text { Design } \\
\text { variable }\end{array}$ & $\begin{array}{l}\text { Lower } \\
\text { bound }\end{array}$ & $\begin{array}{l}\text { Initial } \\
\text { value }\end{array}$ & $\begin{array}{l}\text { Upper } \\
\text { bound }\end{array}$ & $c$ (in (37)) \\
\hline L1 & 7.50 & $\theta_{1}$ & $-90^{\circ}$ & $45^{\circ}$ & $+90^{\circ}$ & 1.1572 \\
L2 & 3.75 & $\theta_{1} / \theta_{2}$ & $-90^{\circ} /-90^{\circ}$ & $45^{\circ} /-45^{\circ}$ & $+90^{\circ} /+90^{\circ}$ & 0.8113 \\
L3 & 2.50 & $\theta_{1} / \theta_{2} / \theta_{3}$ & $-90^{\circ} /-90^{\circ} /-90^{\circ}$ & $45^{\circ} /-45^{\circ} / 45^{\circ}$ & $+90^{\circ} /+90^{\circ} /+90^{\circ}$ & 0.9841 \\
\hline
\end{tabular}

The constraint expressed by (37) is added to the set of constraints of the optimal problem described in (14). Three distinct composite plates, or laminates, for the model of the connecting rod, with one, two and three layers, are considered in this demonstrative example. The characteristics of the composite plates are shown in Table 2.

The optimization problem is normalized, by scaling the design variables and objective function to the unity, in the initial configuration. This avoids numerical difficulties in the optimization procedure that arise from the difference of size of the design variables.

The optimization results are summarized in Table 3 for the three laminate models. The optimization procedure reduces the deformation energy in the order of $90 \%$ and converges to an optimum design, for all laminates. In Fig. 8, the normalized criteria value for several orientations of the lamina in laminate 1 is presented. In this case, only a global optimum exists and this is found in two iterations by the optimization algorithm. Note that for the laminates with two and three layers, several minima exist, and therefore, the initial condi- 
Table 3 Optimization results summary

\begin{tabular}{llll}
\hline & L1 (MFD) & L2 (MFD) & L3 (MFD) \\
\hline Initial & 0.0155818 & 0.011486 & 0.0135897 \\
Optimum & 0.0012237 & 0.001224 & 0.0012520 \\
Reduction & $92.2 \%$ & $89.3 \%$ & $90.8 \%$ \\
Optimum layer & & & \\
orientations & $\left(6.7 \times 10^{-7}\right)^{\circ}$ & $\left(5.8 \times 10^{-4}\right)^{\circ} /\left(6.7 \times 10^{-7}\right)^{\circ}$ & $\left(-6.7 \times 10^{-7}\right)^{\circ} /-18.6^{\circ} /\left(5.87 \times 10^{-4}\right)^{\circ}$ \\
\hline
\end{tabular}

Fig. 8 Variation of the normalized criteria value, for laminate 1 , with the orientation of lamina

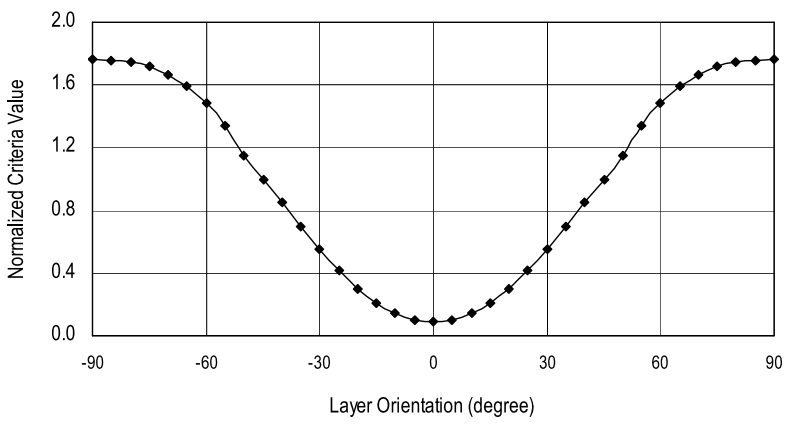

tions of the problem influence which one of them is reached. A good practice is to solve the optimal problem several times with different initial values for the design variables.

The optimum designs for laminate 1 and 2 are coincident, but the optimum design of laminate 3 is different from the other two laminates. This difference is due to the optimum orientation of layer 2 in laminate 3 . In fact, for laminate bending, the external layers are more important than the internal layers. Therefore, the optimization algorithm tends to modify orientations of layers 1 and 3, and to disregard the orientation of layer 2.

It should be noted that in any practical application of composite materials made of laminated layers, there is a tolerance above which it is not possible to control the relative layer orientations. The present technology limits the accuracy at which layer orientations can be set to $5^{\circ}$ increments. This fact more than constituting a constraint in the optimization process enforces the use of optimization methods for discrete variables [61-65], which are not considered in this work.

\subsection{Maximum deformation energy optimization}

The stiffness optimization can be associated with the maximum value of the elastic deformation energy, which is formulated as the min-max optimization described by (19). The extra design variable $\beta \in] 0,1]$ introduced serves as a moving limit for the elastic deformation energy, being its initial value set to 0.8 . This optimal problem has a constraint that is timedependent and, therefore, it is substituted by several time-independent constraints located at the points with higher values of deformation energy in the initial design.

The optimization results are presented in Table 4 for the three laminate models of the rod. The deformation energy responses obtained for this case and for the rod deformation energy of laminate 3 is presented in Fig. 9 for the original design, optimal design with the integral of the deformation energy and optimal solution that minimizes the maximum energy. 
Table 4 Optimization results summary for maximum deformation energy

\begin{tabular}{llll}
\hline & L1 (MFD) & L2 (MFD) & L3 (MFD) \\
\hline Initial & 1.15718 & 0.81113 & 0.984053 \\
Optimum & 0.130411 & 0.101434 & 0.176677 \\
Reduction & $88.7 \%$ & $87.5 \%$ & $82.0 \%$ \\
Optimum layer orientations & $-10.71^{\circ}$ & $-10.92^{\circ} / 10.92^{\circ}$ & $-16.6^{\circ} /-8.8^{\circ} /-16.7^{\circ}$ \\
Optimum $\beta$ & 0.126701 & 0.0980453 & 0.173684 \\
\hline
\end{tabular}

Fig. 9 Deformation energy for the original and optimum models of laminate 3

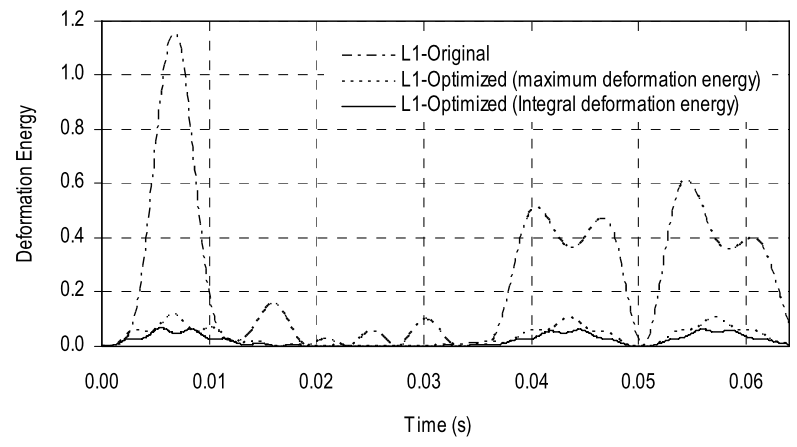

Comparing the two formulations of the optimum design analyzed it is noticed that a high number of function calls is required when using an extra variable that represents a moving limit for the deformation energy. In this case, the method has difficulty in performing the line search procedure and finding the correct step size due to the constraints that are being kept active all the time. In this case, the objective function involving only the integral deformation energy leads to better results.

\subsection{Optimization of local torsion and deformation energy}

Often it is required that the torsion of the plate is minimized while the maximum stiffness of the plate is searched for. A multicriteria formulation is defined using the method of the weighted objectives. The criterion representing the integral of the energy deformation and the torsion of the plate are used in the definition of a new objective function, written as

$$
\Psi_{0}(\boldsymbol{\theta})=w_{1} \int_{t_{0}}^{t_{e}} 2 U_{i}(\boldsymbol{\theta}, t) d t+w_{2} \int_{t_{0}}^{t_{e}}\left(u_{3}^{\prime}{ }^{A}(\boldsymbol{\theta}, t)-u_{3}^{\prime}{ }^{B}(\boldsymbol{\theta}, t)\right)^{2} d t .
$$

Although the values for the weights $w_{1}$ and $w_{2}$ are arbitrary, in what follows, the weight values are chosen to be $w_{1}=0.6$ and $w_{2}=0.4$.

The optimization results for the multicriteria problem defined by (38) are summarized in Table 5 for the three laminate models of the rod. For laminate 1, the optimization terminates when the maximum number of iteration allowed is reached. The evolution of the criteria, depicted by Fig. 10, can be better understood by observing Fig. 11 where the evolution of the objective function value is presented for several lamina orientations of the laminate 1 . For 
Table 5 Multicriteria Optimization results summary

\begin{tabular}{llll}
\hline & L1 (MFD) & L2 (MFD) & L3 (MFD) \\
\hline Initial & 1 & 1 & 1 \\
Optimum & 0.978565 & 0.145417 & 0.632105 \\
Reduction & $2.14 \%$ & $85.45 \%$ & $36.789 \%$ \\
Optimum layer orientations & $49.2^{\circ}$ & $-10.53^{\circ} / 13.37^{\circ}$ & $47.38^{\circ} /-18.25^{\circ} / 47.38^{\circ}$ \\
\hline
\end{tabular}

Fig. 10 Evolution of criterion 1 and 2 during the optimization of laminate 1

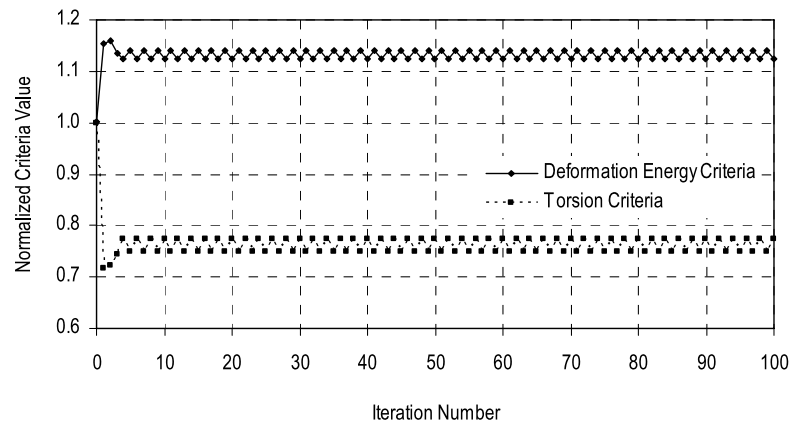

Fig. 11 Objective function for several lamina orientations of the laminate 1

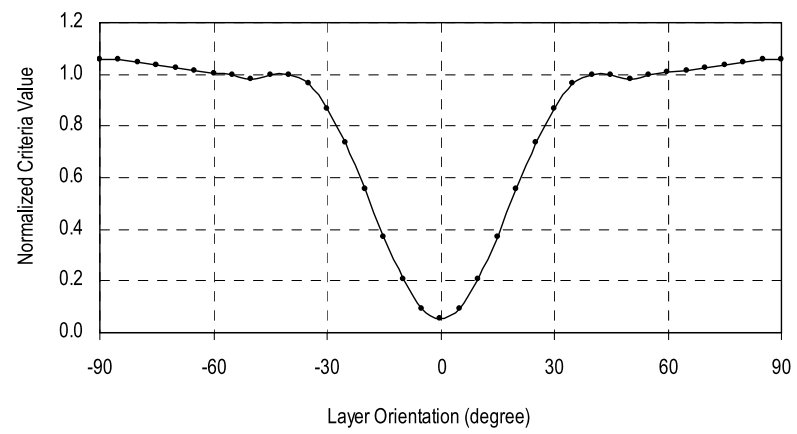

the lamina orientation of $45^{\circ}$ there is a neighborhood with local minima. The optimization method converges to this local minimum, but because only the local properties of sensitivities are taken in consideration, the optimization does not progress any further.

For both cases of laminates 2 and 3, the optimization process only requires four iterations to converge to a minimum. However, care must be used to ensure that such is a global minimum, or at least that it is close to it.

The comments made with the previous study case, concerning the manufacturing tolerances of the layer orientation increments, remain valid for this study case. An industrial application of the results obtained needs to take into account that it is not possible to obtain layer orientation increments below $5^{\circ}$. 


\section{Optimization of the deployment of a satellite antenna}

The methodology for the optimal design of flexible multibody systems is demonstrated through its application to the design of the unfolding process of a satellite antenna, the Synthetic Aperture Radar (SAR), which is a part of the European research satellite ERS-1. Several models for this antenna are found in references by Anantharaman and Hiller [65].

\subsection{Description of the SAR antenna}

During launching the SAR antenna and the solar array are folded, as shown in Fig. 12(a), to be as compact as possible. Upon unfolding the satellite achieves the configuration represented in Fig. 12(b).

The SAR antenna consists in two identical subsystems, each with three coupled planar four-bar links that unfold two panels on each side. The central panel is attached to the main body of the satellite. Each unfolding system has two degree of freedom, driven individually by actuators located in the joints A and B, shown in Fig. 13.

The unfolding process consists in two phases, schematically represented in Fig. 14. In the first phase, the panel 3 is rolled out by a rotational spring-damper-actuator in joint $\mathrm{A}$, while the panel 2 is held down by locking joints $\mathrm{D}$ and E, identified in Fig. 13(a). The second phase begins with the joint A locked being the panels 2 and 3 swung out to the final position by a rotational spring-damped-actuator in joint B, as observed in Fig. 13(a).

The second half of the antenna, which has been omitted in Fig. 13, is unfolded in the same way as the first half shown here. When the complete antenna is deployed, all five panels are aligned in the final configuration. Functional requirements for the final configuration impose that the alignment between panels is obtained within a tolerance smaller than $0.5^{\circ}$.

Fig. 12 Satellite with the SAR antenna in (a) folded and (b) unfolded configurations a)

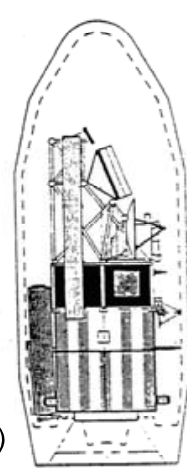

b)

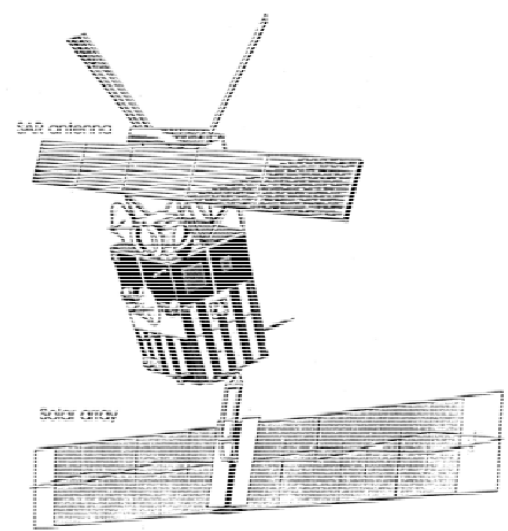

Fig. 13 The SAR antenna: (a) one half unfolded state (b) folded antenna

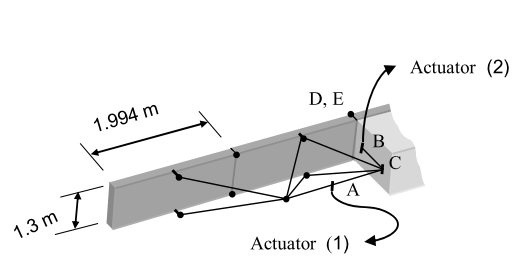

a)

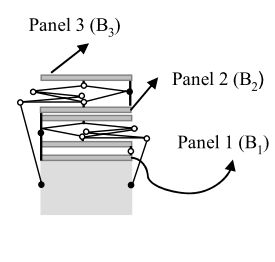

b) 
Fig. 14 Unfolding process of the SAR antenna: (a) first phase (b) second phase
Fig. 15 Multibody model of the SAR antenna

\begin{tabular}{lllll}
\hline & 1st Layer & 2nd Layer & 3rd Layer & 4th Layer \\
\hline Lay-up 1 & $0^{\circ}$ & $0^{\circ}$ & $0^{\circ}$ & $0^{\circ}$ \\
Lay-up 2 & $0^{\circ}$ & $90^{\circ}$ & $90^{\circ}$ & $0^{\circ}$ \\
Thickness (m) & 0.0005 & 0.0005 & 0.0005 & 0.0005 \\
\hline
\end{tabular}

Table 6 Characteristics of the two lay-ups considered for the composite panels

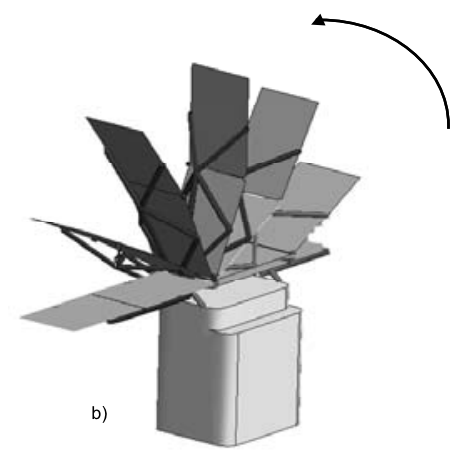

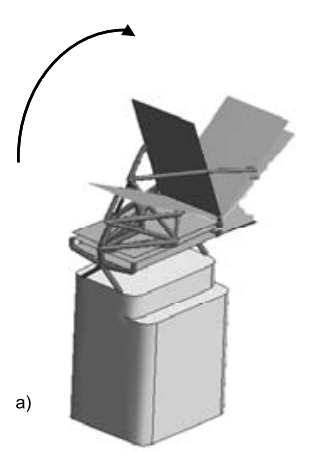
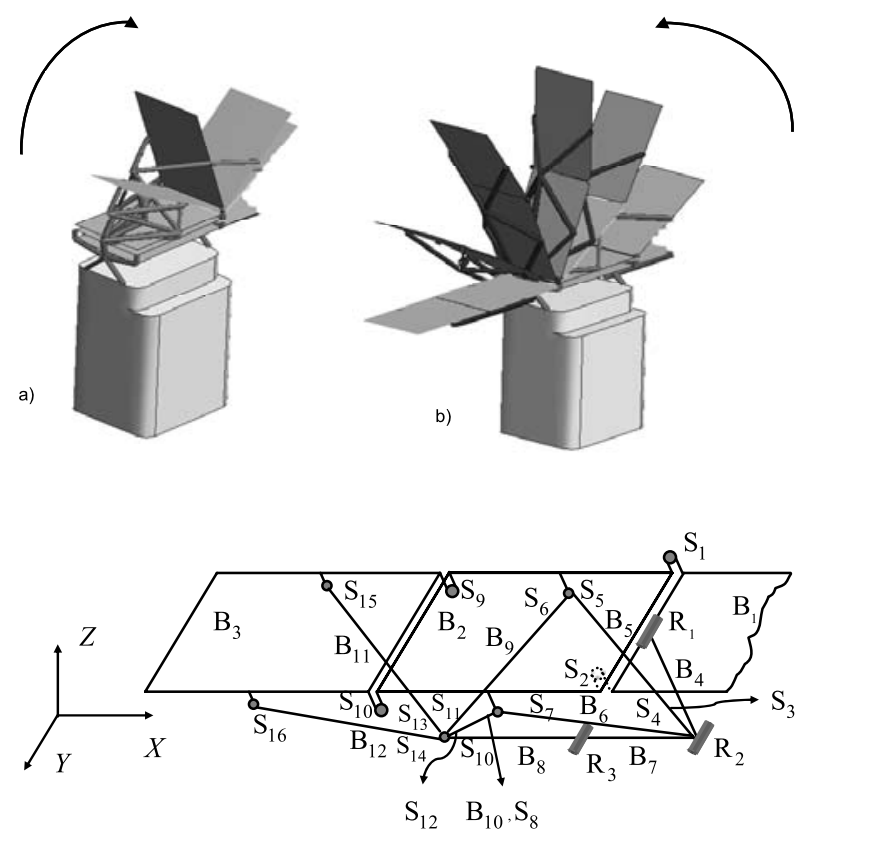


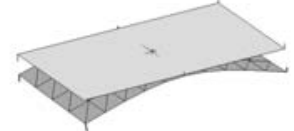

Bending about YY $(\omega=3.3 \mathrm{~Hz})$

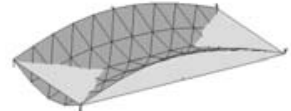

Bending about $\mathrm{XX}(\omega=8.55 \mathrm{~Hz})$

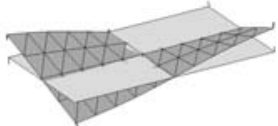

Torsion about XX $(\omega=4.75 \mathrm{~Hz})$

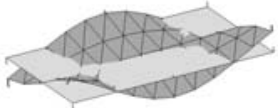

Mixed Bending and Torsion $(\omega=11.6 \mathrm{~Hz})$

Fig. 16 Vibration modes of the panel 2
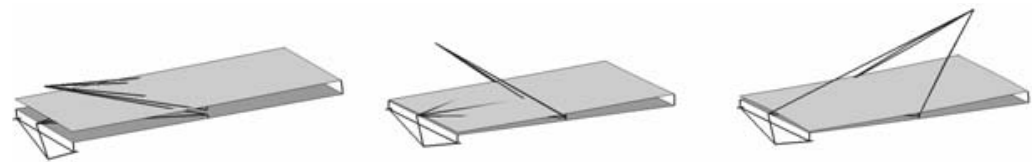

Fig. 17 Configuration of the composite panels with the original damped spring-actuator

\subsection{First phase of the antenna unfolding process}

The actuator that is applied in revolute joint $\mathrm{R}_{3}$ is modeled as a nonlinear spring and damper actuator. The spring-damper-actuator is described by a piecewise-linear characteristic, given by

$$
M(\theta, \dot{\theta})=c \dot{\theta}+ \begin{cases}0.10+9.00(3.12-\theta), & 3.08<\theta \leq 3.12, \\ 0.45+60.41(3.08-\theta), & 3.02<\theta \leq 3.08, \\ 4.03-5.19(3.02-\theta), & 2.63<\theta \leq 3.02, \\ 2.00, & 0.20<\theta \leq 2.63, \\ 10.00 \theta, & -0.20 \leq \theta \leq 0.20, \\ -2.00 & -0.20>\theta\end{cases}
$$

where the damping coefficient used is $c=0.5 \mathrm{~N} \mathrm{~m} \mathrm{~s}$. The actuation law presented here is different from that reported by Anantharaman and Hiller [61], which was used to model the SAR antenna with panels made of isotropic material. When the actuation law used by Anantharaman and Hiller is used for the composite flexible models, the satellite antenna is driven to a different equilibrium state than that obtained in the rigid model. The trusses connected to actuator quickly reach their equilibrium, but the panel 3 hardly moves because the unfolding trusses break through the panel, as represented in Fig. 17. This behavior is clearly unfeasible because, before that happens, the contact between trusses and panels would take place. Therefore, the reported results show that due to the deformations of the trusses the undesirable contacts between trusses and panels are possible if the high torques associated to the original actuator have been maintained. Consequently, the solution is to apply an actuation law that prevents such contact.

When using composite material models, the problem of the first phase of the unfolding process increases in importance not only because the bending of the panels is significant, but also because the torsional modes come in play. In Fig. 18, the variation of the actuator angle during the simulation period for the composite models is presented.

Figure 18 shows that the two models lead to simulation results with a similar behavior. However, it is observed that after the equilibrium positions are reached for both models, in 
Fig. 18 Actuator angle for the flexible antenna in the first phase
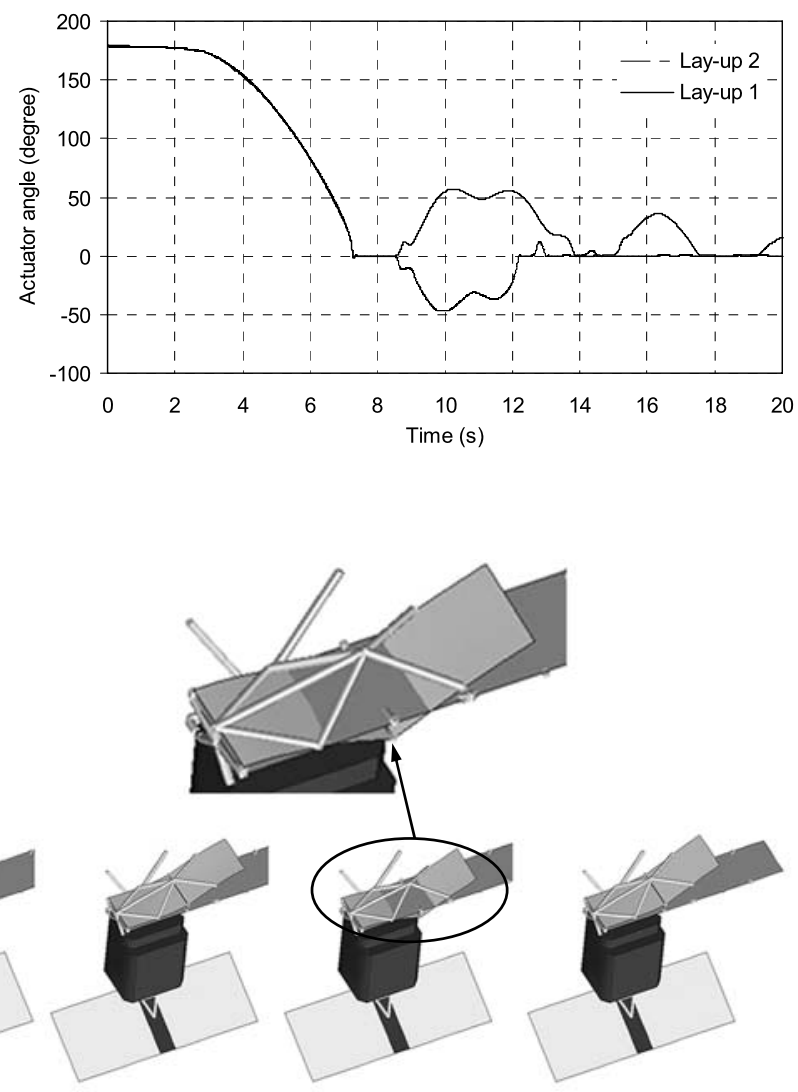

a)
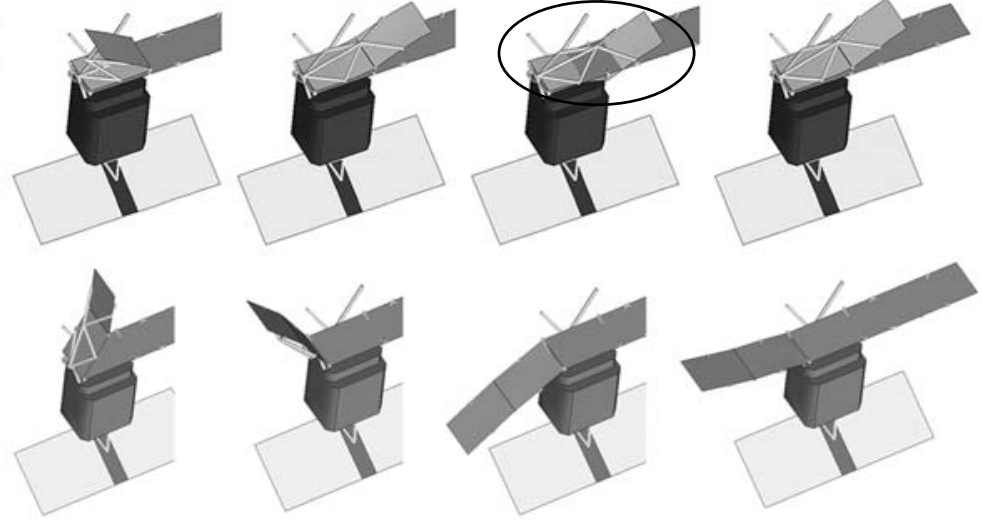

b)

Fig. 19 Configuration of the antenna unfolded process: (a) in the first phase (b) in the second phase

the period from 7 to $8 \mathrm{~s}$, the direction of rotation of the truss members of the panels made with the lay-up 1 is opposite to that of the model made with lay-up 2. This discrepancy can result from the difference between the vibration modes of the models. In fact, the lay-up 1 has no layers with the $90^{\circ}$ orientation, thus the stiffness of this model in the Y direction is smaller than that observed with lay-up 2. The same difference in stiffness is also visible in the direction $\mathrm{X}$ of the lay-up 1 .

The flexible model of the satellite antenna predicts interference between panel 2 and panel 1, which is attached to the flexible base, when bodies $\mathrm{B}_{7}$ and $\mathrm{B}_{8}$ get aligned as shown in the fourth frame of Fig. 19(a). This can be perceived as flaw in the design of the unfolding process of the satellite. 


\subsection{Optimization of the SAR antenna}

As demonstrated with the application to the slider crank, the flexibility of the multibody systems components made of composite materials is very sensitive to the layer orientation of the lay-ups that form the laminate. In order to get a stiffer antenna, the optimization problem is formulated as the minimization of the deformation energy of the panels defined by (21) and rewritten here for panels 2 and 3 as

$$
\Psi_{0}=\int_{t_{0}=0}^{t_{e}=3} U_{2}\left(\mathbf{u}_{2}^{\prime}, t\right) d t+\int_{t_{0}=0}^{t_{e}=3} U_{3}\left(\mathbf{u}_{3}^{\prime}, t\right) d t .
$$

The simulation period that is crucial for a correct deployment is $t_{i}=0 \mathrm{~s} \leq t \leq t_{e}=3 \mathrm{~s}$. In order to avoid that at a given instant the deformation of the panel exceeds predefined limits, the maximum value of the deformation energy, in each panel, is constrained to be

$$
\Psi_{i}(\boldsymbol{\theta}) \leq c_{i} ; \quad i=2,3
$$

with the values $c_{i}$ defined as the maximum value of deformation energy, in each panel, observed in each initial design.

All material models considered herein are symmetric laminates being the number of layers fixed in each of them. Moreover, the laminate model used to model the material properties of panels 2 and 3 is the same. The two design variables used in the optimization process correspond to the orientation of the layers that make up the laminate used to model the panels. The initial design of laminate used in the panels is defined in Table 7. The optimization method used is the Modified Method of Feasible Directions (MFD).

The optimization results are summarized in Table 8 for the flexible multibody of the antenna. Figure 20 presents the evolution of the objective function for the antenna flexible multibody model, being the progress of the design variables shown in Fig. 21.

For this optimization scenario it is possible verify that the optimization procedure converges very fast to the optimum solution, reducing the deformation energy in the order of 95\%, as seen in Fig. 20. The largest variation of the design variables that occurred is associated with the outside layers of the laminate, as observed in Fig. 21.

The evolution of the deformation energy of panels 2 and 3, for the initial and optimized designs, are displayed in Figs. 22 and 23, respectively. It is visible in these figures that the major contribution to the reduction of the deformation energy is provided by panel 2 .

Table 7 Design variables for the satellite on the second optimization scenario

\begin{tabular}{lllll}
\hline Panels & Design variable & Lower bound & Initial value & Upper bound \\
\hline $2=3$ & $\theta_{1} / \theta_{2} / \theta_{2} / \theta_{1}$ & $-90^{\circ} /-90^{\circ} /-90^{\circ} /-90^{\circ}$ & $55^{\circ} /-55^{\circ} /-55^{\circ} / 55^{\circ}$ & $+90^{\circ} /+90^{\circ} /+90^{\circ} /+90^{\circ}$ \\
\hline
\end{tabular}

Table 8 Summary of the optimization results of the satellite on the second optimization scenario

$\begin{array}{ll}\text { Optimum layer orientations } & 1.06^{\circ} /-47^{\circ} /-47^{\circ} / 1.06^{\circ} \\ \text { Initial objective function } & 0.0219814 \\ \text { Optimum objective function } & 0.00097180 \\ \text { Reduction of objective function } & 95.6 \%\end{array}$


Fig. 20 Objective function for the second optimization scenario

Fig. 21 Evolution of the design variables in the second optimization scenario
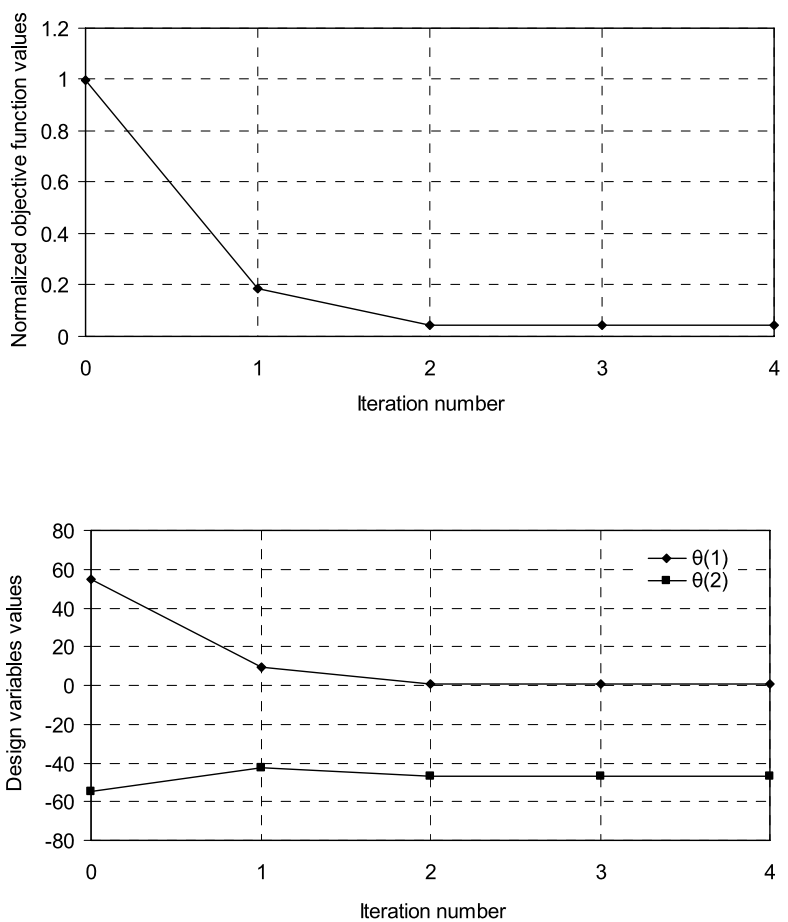

Fig. 22 Deformation energy for the initial and optimal model of panel 2

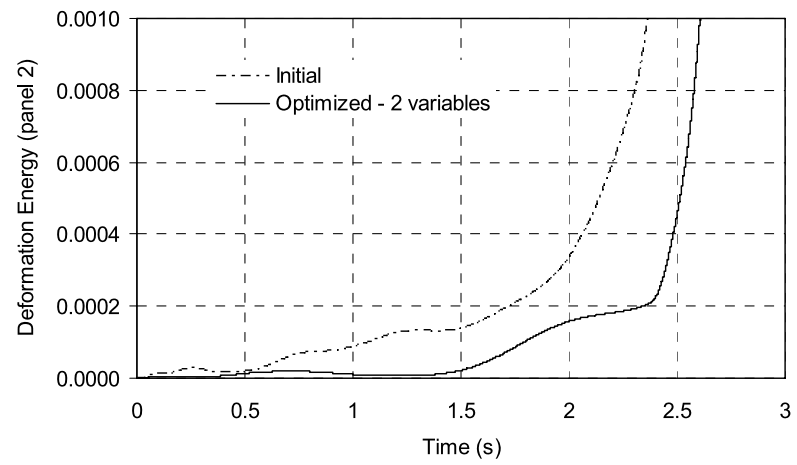

Another aspect of the deployment requiring analysis concerns the interference detected in the first phase of the deployment for the initial design, illustrated in Fig. 19. Figures 24 and 25 show the normal displacement of point $B$, in panel 2 , and of node $D$, in panel 3 for 20 seconds of simulation. There it is possible to observe that the normal displacements of the panels, with respect to the body fixed frame, are greatly reduced, favoring in this form not only the possibility of interference between panels but also their torsion.

In Fig. 26, the actuator angle history during the first phase of the unfolding antenna is presented for the original and the optimum designs. It is clear that both designs lead to similar end alignments of the panels. 
Fig. 23 Deformation energy for the initial and optimal model of panel

Fig. 24 Normal displacements of node $B$ in panel 2 for the initial and optimal laminates
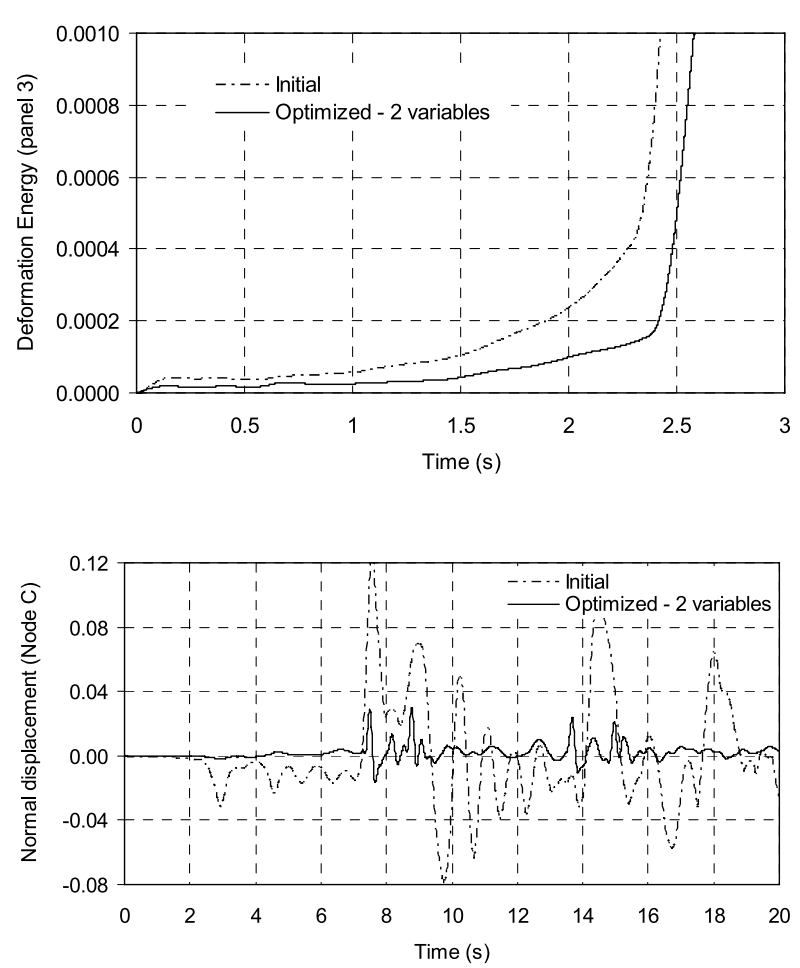

Fig. 25 Normal displacements of node $D$ in panel 3 for the initial and optimal laminates

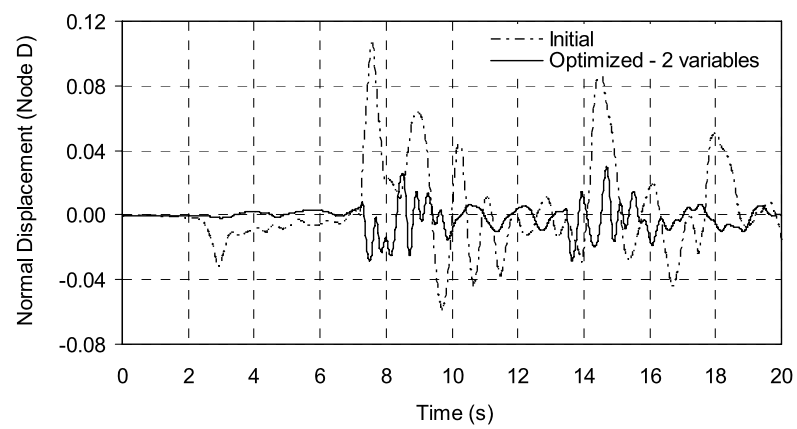

\section{Conclusions}

A general method for the design optimization of flexible multibody systems made of composite materials has been presented in this work, with emphasis on a flexible multibody formulation involving composite materials and on the efficient evaluation of the sensitivities. For the type of problems demonstrated here, it can be concluded that the use of numeric sensitivities is not recommended, not only because there is no clear rule on the size of the perturbation that can be used, but also because not always do they allow the evaluation of correct sensitivities. For flexible multibody systems with composite materials, in which the design variables are the layer orientations, there is no alternative to the analytical sensitivities. The use of automatic differentiation for the derivation of the system sensitivities 
Fig. 26 Actuator angle for the initial and optimal laminates during the first phase

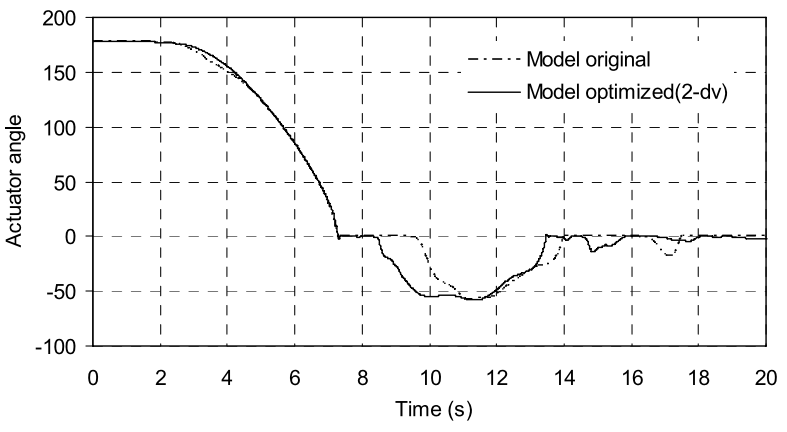

demonstrated to be an accurate and efficient tool to obtain the computer code for the analytical sensitivities, provided that some of its parts are used with caution. The sensitivities of the modes of vibration, in particular, cannot be handled by the code generated by the automatic differentiation tool as choices in methodologies have to be made if repeated modes exist in any of the structural components. Moreover, it has been concluded that for the sensitivity of the modes of vibration only the updated mode approach is reliable. The demonstration of the methodology with a simple slider-crank mechanism with a flexible connecting rod allows to show not only the merits and drawbacks of different aspects of the methodology proposed in this work but also to identify the suitable objective functions that aim at the stiffening of the system components made of composite materials. A suitable objective function is the integral of the deformation energy with the addition of an extra constraint to the optimization procedure to prevent that the deformation energy exceeds a predefined value at any time. The methodology was finally applied to a complex system represented by the multibody model of the SAR antenna. The results demonstrate a feasible design for the antenna in which no interference between panels is observed as a result of the application of the methodology proposed here.

Acknowledgements We thank Professors M. Hiller and A. Kecskemethy for providing the data necessary to model the SAR antenna. We would also like to thank Professors O. Bauchau and D. Hodges for making the source code of VABS available to our work.

\section{References}

1. Belytschko, T., Liu, W.K., Moran, B.: Nonlinear Finite Elements for Continua and Structures. Wiley, Chichester (2000)

2. Crisfield, M.A.: Nonlinear Finite Element Analysis of Solids and Structures, vol. 2: Advanced Topics. Wiley, Chichester (1997)

3. Géradin, M., Cardona, A.: Flexible Multibody Dynamics: A Finite Element Approach. Wiley, Chichester (2001)

4. Shabana, A.A.: Dynamics of Multibody Systems. Wiley, New York (1989)

5. Ambrósio, J.: Dynamics of structures undergoing gross motion and nonlinear deformations: a multibody approach. Comput. Struct. 59(6), 1001-1012 (1996)

6. Ambrósio, J., Kleiber, M. (eds.): Computational Aspects of Nonlinear Structural Systems With Large Rigid Body Motion. IOS, Amsterdam (2001)

7. Nikravesh, P.: Computer-Aided Analysis of Mechanical Systems. Prentice-Hall, Englewood Cliffs (1988)

8. Ambrósio, J., Gonçalves, J.: Complex flexible multibody systems with application to vehicle dynamics. Multibody Syst. Dyn. 6(2), 163-182 (2001)

9. Kane, T., Ryan, R., Banerjee, A.: Dynamics of a cantilever beam attached to a moving base. AIAA J. Guid. Control Dyn. 10, 139-151 (1987) 
10. Garcia de Jalon, J., Bayo, E.: Kinematic and Dynamic Simulation of Mechanical Systems-The RealTime Challenge. Springer, Berlin (1994)

11. Shabana, A.: Definition of the slopes and the finite element absolute nodal coordinate formulation. Multibody Syst. Dyn. 1, 339-348 (1997)

12. Dmitrochenko, O., Yoo, W.-S., Pogorelov, D.: Helicoseir as shape of a rotating string (I): 2D theory and simulation using ANCF. Multibody Syst. Dyn. 15(2), 135-158 (2006)

13. Gerstmayr, J., Schöberl, J.: A 3D finite element method for flexible multibody systems. Multibody Syst. Dyn. 15(4), 305-320 (2006)

14. Shabana, A.A., Wehage, R.A.: A coordinate reduction technique for transient analysis of spatial structures with large angular rotations. J. Struct. Mech. 11, 401-431 (1989)

15. Craig, R.R. Jr., Bampton, M.C.C.: Coupling of substructures for dynamic analyses. AIAA J. 6(7), 13131319 (1968)

16. Nikravesh, P., Lin, Y.-S.: Use of principal axes as the floating reference frame for a moving deformable body. Multibody Syst. Dyn. 13(2), 211-231 (2005)

17. Pereira, M., Proença, P.: Dynamic analysis of spatial flexible multibody systems using joint coordinates. Int. J. Numer. Methods Eng. 32, 1799-1812 (1991)

18. Yoo, W., Haug, E.: Dynamics of flexible mechanical systems using vibration and static correction modes. J. Mech. Transm. Autom. Des. 108, 315-322 (1986)

19. Liu, J.-F., Yang, J., Abdel-Malek, K.: Dynamics analysis of linear elastic planar mechanisms. Multibody Syst. Dyn. 17(1), 1-25 (2007)

20. Ambrósio, J., Ravn, P.: Elastodynamics of multibody systems using generalized inertial coordinates and structural damping. Mech. Struct. Mach. 25, 201-219 (1997)

21. Lehner, M., Eberhard, P.: On the use of moment-matching to build reduced order models in flexible multibody dynamics. Multibody Syst. Dyn. 16(2), 191-211 (2006)

22. Pombo, J., Ambrósio, J.: General spatial curve joint for rail guided vehicles kinematics and dynamics. Multibody Syst. Dyn. 9(3), 237-264 (2003)

23. Angeles, J., Lopez-Cajun, C.S.: Optimization of CAM Mechanisms. Springer, Dordrecht (1991)

24. Ambrósio, J.: Efficient kinematic joints descriptions for flexible multibody systems experiencing linear and non-linear deformations. Int. J. Numer. Methods Eng. 56, 1771-1793 (2003)

25. Gonçalves, J., Ambrósio, J.: Advanced modeling of flexible multibody dynamics using virtual bodies. Comput. Assist. Mech. Eng. Sci. 9(3), 373-390 (2002)

26. Muñoz, J., Jelenic, G.: Sliding joints in 3D beams: conserving algorithms using the master-slave approach. Multibody Syst. Dyn. 16(3), 237-261 (2006)

27. Cesnik, C., Hodges, D.: VABS: a new concept for composite rotor blade cross-sectional modeling. J. Am. Helicopter Soc. 42(1), 27-38 (1997)

28. Augusta Neto, M., Ambrósio, J., Leal, R.: Flexible multibody systems using composite materials components. Multibody Syst. Dyn. 12(4), 363-405 (2004)

29. Bauchau, O., Hodges, D.: Analysis of nonlinear multibody systems with elastic couplings. Multibody Syst. Dyn. 3, 163-188 (1999)

30. Augusta Neto, M., Ambrósio, J., Leal, R.: Composite materials in flexible computational multibody dynamics. Comput. Methods Appl. Mech. Eng. 195, 6860-6873 (2006)

31. Venkataraman, S., Haftka, R.: Optimization of composite panels-a review. In: Proc. of the 14th Annual Technical Conference of the American Society of Composites, Dayton, OH, 27-29 September 1999

32. Eberhard, P., Dignath, F., Kübler, L.: Parallel evolutionary optimization of multibody systems with application to railway dynamics. Multibody Syst. Dyn. 9, 143-164 (2003)

33. Eberhard, P., Schiehlen, W., Bestle, D.: Some advantages of Stochastic methods in multicriteria optimization of multibody systems. Arch. Appl. Mech. 69, 543-554 (1999)

34. Adelman, H.M., Haftka, R.T.: Sensitivity analysis of discrete structural systems. AIAA J. 24, 823-832 (1986)

35. Greene, W., Haftka, R.: Computational aspects of sensitivity calculations in transient structural analysis. Comput. Struct. 32(2), 433-443 (1989)

36. Haftka, R., Gürdal, Z.: Elements of Structural Optimization. Kluwer Academic, Dordrecht (1992)

37. Dias, J., Pereira, M.: Sensitivity analysis of rigid-flexible multibody systems. Multibody Syst. Dyn. 1, 303-322 (1997)

38. Dias, J.: Análise de sensibilidades e optimização de sistemas mecânicos rígido-flexíveis (Sensitivity analysis and optimization of rigid-flexible mechanical systems). PhD thesis, Instituto Superior Técnico de Lisboa, Technical University of Lisbon, Lisbon, Portugal (1999)

39. Bischof, C., Carle, A., Corliss, G., Griewank, A., Hovland, P.: ADIFOR: generating derivative codes from Fortran programs. Sci. Program. 1(1), 11-29 (1992)

40. Bischof, C., Carle, A., Khademi, P., Mauer, A.: ADIFOR 2.0: automatic differentiation of Fortran 77 programs. IEEE Comput. Sci. Eng. 3(3), 18-32 (1996) 
41. Venkataraman, S., Haftka, R.: Structural optimization: what has Moore's law done for us? In: 43rd AIAA/ASME/ASCE/AHS/ASC Structures, Structural Dynamics and Materials Conference, Denver, CO, USA, 22-25 April 2002

42. Vanderplaats, G.: DOT-Design Optimization Tools, Version 3.0. VMA Engineering, Colorado Springs (1992)

43. Cook, R.: Concepts and Applications of Finite Element Analysis. 2nd edn. Wileyand, New York (1987)

44. Duff, A., Erisman, Reid, J.: Direct Methods for Sparse Matrices. Clarendon, Oxford (1986)

45. Cavin, R., Dusto, A.: Hamilton's principle: finite element method and flexible body dynamics. AIAA J. 15(12), 1684-1690 (1977)

46. Augusta Neto, M.: Optimization of flexible multibody systems with composite materials. PhD thesis, Mech. Engng Dept., Univ. of Coimbra, Coimbra, Portugal (2005)

47. Cesnik, C., Hodges, D.: VABS: a new concept for composite rotor blade cross-sectional modeling. J. Am. Helicopter Soc. 42(1), 27-38 (1997)

48. Popescu, B., Hodges, D.: On asymptotically correct Timoshenko-like anisotropic beam theory. Int. J. Solids Struct. 37, 535-558 (2000)

49. Bauchau, O., Hodges, D.: Analysis of nonlinear multibody systems with elastic couplings. Multibody Syst. Dyn. 3, 163-188 (1999)

50. Chang, C., Nikravesh, P.: Optimal design of mechanical systems with constraint violation stabilization method. J. Mech. Transm. Autom. Des. 107, 493-498 (1985)

51. Kim, M., Choi, D.: A new approach to the min-max dynamic response optimization. In: Bestle, D., Schiehlen, W. (eds.) IUTAM-Symposium on Optimization of Mechanical Systems, pp. 65-72. Kluwer Academic, Dordrecht (1996)

52. Gonçalves, J., Ambrósio, J.: Road vehicle modeling requirements for optimization of ride and handling. Multibody Syst. Dyn. 13(1), 3-23 (2005)

53. Haug, E., Arora, J.: Applied Optimal Design. Wiley, New York (1979)

54. Hsieh, C., Arora, J.: Design sensitivity analysis and optimization of dynamic response. Comput. Methods Appl. Mech. Eng. 43, 195-219 (1984)

55. Grandhi, R., Haftka, R., Watson, L.: Design-oriented identification of critical times in transient response. AIAA J. 24, 649-656 (1986)

56. He, Y., McPhee, J.: Multidisciplinary optimization of multibody systems with application to the design of rail vehicles. Multibody Syst. Dyn. 14(2), 111-135 (2005)

57. Eberhard, P., Bischof, C.: Automatic differentiation of numerical integration algorithms. Math. Comput. 68, 717-731 (1999)

58. Nelson, R.B.: Simplified calculation of eigenvector derivatives. AIAA J. 14(9), 1201-1205 (1976)

59. Dailey, L.: Eigenvector derivatives with repeated eigenvalues. AIAA J. 27(4), 486-491 (1989)

60. Duran, M.A., Grossmann, I.E.: An outer approximation algorithm for a class of mixed-integer nonlinear programs. Math. Program. 36, 307-339 (1986)

61. Geoffrion, A.M.: Generalized Benders decomposition. J. Optim. Theory Appl. 10(4), 237-260 (1972)

62. Gupta, O.K., Ravindran, A.: Branch and bound experiments in convex nonlinear integer programming. Manag. Sci. 31, 1533-1546 (1985)

63. Tawarmalani, M., Sahinidis, N.V.: Global optimization of mixed-integer nonlinear programs: a theoretical and computational study. Math. Program. 99(3), 563-591 (2004)

64. Ng, K.-M.: A continuation approach for solving nonlinear optimization problems with discrete variables. PhD dissertation, Stanford University, Stanford, CA (2002)

65. Anantharaman, M., Hiller, M.: Numerical simulation of mechanical systems using methods for differential-algebraic equations. Int. J. Numer. Methods Eng. 32, 1531-1542 (1991) 